\title{
APOEe4.Trem2*R47H Mice Show Changes in Alzheimer's Disease-Relevant Processes in the Absence of Amyloid Plaques
}

Kevin P. Kotredes ( $\nabla$ kevin.kotredes@jax.org )

The Jackson Laboratory

Adrian Oblak

Stark Neuroscience

Ravi S. Pandey

The Jackson Laboratory

Peter Bor-Chian Lin

Stark Neuroscience

Dylan Garceau

The Jackson Laboratory

Harriet Williams

The Jackson Laboratory

Asli Uyar

The Jackson Laboratory

Rita O'Rourke

The Jackson Laboratory

Sarah O'Rourke

The Jackson Laboratory

Cynthia Ingraham

Stark Neuroscience

Daria Bednarycek

The Jackson Laboratory

Melisa Belanger

The Jackson Laboratory

Zackary Cope

University of Pittsburgh

Kate E . Foley

The Jackson Laboratory

Benjamin A Logsdon

Sage Bionetworks

Lara M . Mangravite 
Sage Bionetworks

Stacey J . Sukoff Rizzo

University of Pittsburgh

Paul R . Territo

Stark Neuroscience

Gregory W . Carter

The Jackson Laboratory

\section{Michael Sasner}

The Jackson Laboratory

Bruce T . Lamb

Stark Neuroscience

Gareth R. Howell

The Jackson Laboratory

\section{Research Article}

Keywords: Late-onset Alzheimer's disease, disease progression, Model Organism Development, risk factors interact

Posted Date: June 15th, 2021

DOI: https://doi.org/10.21203/rs.3.rs-580913/v1

License: (c) (1) This work is licensed under a Creative Commons Attribution 4.0 International License.

Read Full License 
5 Kevin P. Kotredes ${ }^{1}$, Adrian Oblak ${ }^{2}$, Ravi S. Pandey ${ }^{1}$, Peter Bor-Chian Lin², Dylan Garceau ${ }^{1}$, Harriet

6 Williams ${ }^{1}$, Asli Uyar ${ }^{1}$, Rita O’Rourke ${ }^{1}$, Sarah O'Rourke ${ }^{1}$, Cynthia Ingraham², Daria Bednarycek ${ }^{1}$, Melisa

7 Belanger ${ }^{1}$, Zackary Cope ${ }^{3}$, Kate E. Foley ${ }^{1}$, Benjamin A Logsdon ${ }^{4}$, Lara M. Mangravite ${ }^{4}$, Stacey J. Sukoff

8 Rizzo $^{3}$, Paul R. Territo ${ }^{2}$, Gregory W. Carter ${ }^{1}$, Michael Sasner $^{1}$, Bruce T. Lamb ${ }^{2 \dagger}$ and Gareth R. Howell ${ }^{1 \dagger}$.

9

$10{ }^{1}$ The Jackson Laboratory, Bar Harbor, ME, USA.

112 Stark Neuroscience, Indianapolis, IN, USA.

$12{ }^{3}$ University of Pittsburgh, Pittsburgh, PA, USA.

$13 \quad{ }^{4}$ Sage Bionetworks, Seattle, WA, USA.

14

15 †Correspondence: gareth.howell@jax.org and btlamb@iu.edu

16 


\section{$1 \quad$ ABSTRACT}

Late-onset Alzheimer's disease (LOAD) is the most common human neurodegenerative disease.

3 Legacy amyloidogenic mouse models have been useful for understanding disease progression, however

4 in the face of failing human trials more focus on disease translation with new mouse strains that better 5 model human Alzheimer's disease (AD) is required. MODEL-AD (Model Organism Development and

6 Evaluation for Late-onset $A D$ ) groups are identifying and integrating disease-relevant, humanized gene

7 sequences from public databases to create more translatable mouse models for therapy development. Mice 8 expressing strong genetic risk factors for LOAD, $A P O E \varepsilon 4$ and $T r e m 2^{*} R 47 H$, were extensively aged and 9 assayed using a multi-disciplined phenotyping approach associated with and relative to human $A D$ 10 pathology. Behavioral, transcriptomic, metabolic, and neuropathological assays identified sex and age as 11 the main sources of variation between genotypes including age-specific enrichment of AD-related 12 processes in the absence of mouse amyloid plaque formation. These data provide an important, baseline 13 understanding of the individual effects and interaction between two strong genetic risk factors for LOAD.

14 These two alleles together form a sensitized, background strain (B6.APOE4.Trem2*R47H, which we have 15 termed 'LOAD1') necessary to examine how important underlying risk factors interact with any subsequent 16 genetic or environmental cues to drive pathology.

\section{INTRODUCTION}

Alzheimer's disease $(A D)$ is the most common form of dementia, currently affecting 5.8 million

21 patients in the United States (1), 95\% of which are late-onset (LOAD) (2). There are no approved

22 interventions to delay or reverse AD-related neurodegeneration (3). LOAD is a heterogeneous disease

23 defined by a few widely accepted hallmarks: extracellular amyloid plaques, intracellular tau tangles,

24 vascular disfunction, immune activation, synapse loss, neuron death, and cognitive decline (4). Dissecting

25 the etiology of these properties in animal models has provided key insights into our understanding of the

26 disease and propose strategies to treat $A D$, however human clinical trials of resultant therapeutics have not

27 yielded an approvable drug. 
In 2015, the National Institutes on Aging (NIA) aimed to accelerate the AD drug testing pipeline by promoting the design of more predictive preclinical studies that better translate to human disease (3).

3 Current popular animal models of $A D$ rely on the expression of transgenic alleles to promote the 4 aggregation of amyloid plaques or neurofibrillary tangles as drivers of subsequent disease processes, notably during adolescence and early adulthood (5). Therefore, these overexpression models do not fully capitulate the breadth of the disease observed in human patients, particularly elderly populations; many underlying biological pathways affected in human disease are not observed in these mice (6). From this need the MODEL-AD (Model Organism Development and Evaluation for Late-onset AD) consortium was established to provide the research community with the next generation of AD animal models (7). In an effort to design and validate novel mouse models of LOAD that better mimic human disease, we opted to create knock-in humanized coding and non-coding LOAD risk variants expressed at endogenous levels. To

12 implement these considerations in the development of a new generation of preclinical animal models, 13 candidate humanized gene variants or SNPs from a number of public data repositories (ADSP, AMP-AD, 14 ADGC, ADNI, ROS/MAP, IGAP, M²OVE-AD, Resilience-AD, ACT) are being identified and engineered into 15 existing mouse genes under endogenous promoters (8). As a heterogenous disease, it is unlikely that a 16 single genetic alteration will promote the complex set of endophenotypes observed in humans. Rather, a 17 combination of genetic and/or environmental risk factors are likely needed to phenocopy human disease 18 and a better understanding of the effects of the complex interrelationships between risk factors is required 19 to identify prospective therapeutic avenues. To this end, new mouse strains and conditions better replicating 20 human LOAD are needed to improve the clinical translation of mouse therapies in human patients.

To date, approximately 40 loci have been identified through genetic and genome wide association

22 studies that increase risk for $A D(9-11)$. The strongest of these risk factors include the $\varepsilon 4$ allele of 23 apolipoprotein $\mathrm{E}(A P O E)$ and point mutations in triggering receptor expressed on myeloid cells 2 (TREM2) $24 \operatorname{locus}(2,6,10-13)$.

$A P O E$, the strongest genetic determinant of LOAD risk, has been the focus of extensive 26 investigation for many years (14-16), including the development of multiple mouse models (17-20). APOE 27 is important in lipoprotein metabolism and immunoregulation strongly associated with cardiovascular and 28 Alzheimer's disease $(2,6,8,10-13,21)$. Three isoforms of $A P O E$ are expressed in the human population: 
$A P O E \varepsilon 2, A P O E \varepsilon 3$, and $A P O E \varepsilon 4$ which confer increasing risk of $L O A D$, respectively. Compared to $A P O E \varepsilon 3$ carriers, $\varepsilon 4$ isoform of $A P O E$ increases $A D$ risk and decreases age of diagnoses $(22,23)$. The three isoforms differ by one amino acid each at positions 112 and 158 that has profound effects on their functions:

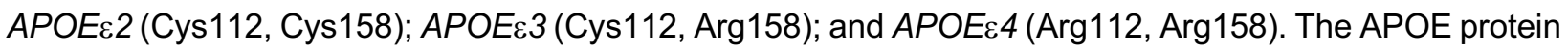

5 has been shown to stimulate binding, transport, and metabolism of lipoproteins, major cholesterol transporters in the central nervous system (CNS) resulting in hypocholesteremia, tight junction failure, and vascular dysregulation $(24,25)$. Additionally, reports of mice carrying human $A P O E_{\varepsilon} 4$-targeted replacement allele show evidence of blood-brain barrier (BBB) leakiness $(26,27)$, immune alterations (28-31), synaptic disfunction (19), and behavior deficits (32). Most importantly, APOE is also implicated in beta-amyloid and tau clearance $(33,34)$. However, many of the aspects of LOAD are not recapitulated upon expression of

$11 A P O E_{\varepsilon} 4$ alone, including formation of beta-amyloid plaques and neurofibrillary tangles (32).

TREM2 encodes a member of a receptor signaling complex with TYRO protein tyrosine kinase

13 binding (TYROBP) protein, which activates microglia, macrophages, and dendritic cells during damage and

14 immune responses (35-37) functioning in processes like debris clearance (38) and amyloid plaque 15 response (39-41). Single nucleotide polymorphisms (SNP) found in TREM2 have been shown to regulate 16 microglial function $(36,42,43)$, the most widely studied being the R47H missense mutation in exon 2 . The

$17 T R E M 2^{*} R 47 \mathrm{H}$ mutation triples the carrier's likelihood of Alzheimer disease $(2,6,10-13)$. The increased risk

18 is suggested to be, in part, the result of decreases in the microglial receptor's interactions with ligands

19 (phospholipids, APOE, and beta-amyloid) yielding chronic dysfunction in microglial phagocytosis and 20 inflammatory pathways $(8,35,42)$.

21 Here we describe the application of a multi-faceted, cross-sectional phenotyping approach 22 developed by MODEL-AD that included biometrics, behavioral assays, transcriptomics, neuroimaging, and 23 immunohistochemistry to assess AD-relevant phenotypes in mice expressing combinations of humanized 24 APOE\&4 and $\operatorname{Trem}^{*} R 47 \mathrm{H}$ alleles.

\section{RESULTS}

\section{Experimental cohorts}


To decipher how these two strong risk factors drive AD-relevant phenotypes, we created a double homozygous B6.APOE4.Trem2*R47H model, accompanied by single genotype controls, on a C57BL/6J (B6) background (Table 1). In appreciation of sexual dimorphism observed in human aging and disease, cohorts of males and females were established for phenotyping at 4-, 8-, 12- and 24-months using a crosssectional design. To determine whether the LOAD risk variants $A P O E_{\varepsilon} 4, T r e m 2^{*} R 47 H$, or the combination produced in vivo phenotypes independent from normal healthy aging, a comprehensive cross-sectional phenotyping battery was conducted and included in vivo frailty assessments, metabolic screening, microbiome sampling, biomarker evaluation, behavioral phenotyping, and in vivo imaging. Postmortem brain tissue was further examined for transcriptomic and neuropathological indications of disease. All accumulated data sets and observations are disseminated for public availability (44).

\section{Biometric profiles of APOE4.Trem $2^{*} R 47 H$ mice change with age}

Comparison of biometric data from young (4 months) and aged mice (24 months), comprised of

15 characteristics (Figure 1 $A, B)$ with inverse correlations in body temperature (Supplemental Figure 1) and

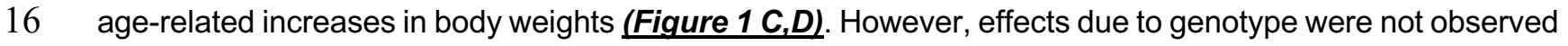

17 overall (see also Supplemental Table 1). Statistical analyses were constrained to contrasting only cohorts

18 sharing littermates. To determine the effects of $A P O E \varepsilon 4$ and $T r e m 2{ }^{*} R 47 H$ on mouse metabolome, terminal

19 non-fasted blood plasma levels of metabolites were measured. Homozygous expression of humanized

$20 A P O E_{\varepsilon 4}$ resulted in a significant decrease of non-fasted serum low-density lipoproteins (LDL) in the

21 absence of corresponding decreases in total cholesterol (Figure $1 \mathbf{E}-\mathbf{H}$ ). Expected aging-specific-effects

22 included decreases in glucose and triglyceride levels in both sexes independent of genotypes (Figure 1I-

23 LL). Other measurements and metabolic analytes included in our panel were unchanged between sex, age,

24 and genotype cohorts (Supplemental Figure 1 C-F and Supplemental Table 2). Additional cohorts,

25 investigating $A P O E_{\varepsilon} 4$ allele alone compared with littermate B6 controls, were aged to 12 months.

26 Consistent with initial aging related phenotypes in the 4- and 24-month cohorts, there was an expected

27 increase in cumulative frailty scores, reductions in core body temperature and increase in body weight in

28 both sexes with no genotype-driven differences observed (Supplemental Figure 1 G-I). 
Age is the strongest determinant of performance by APOE4.Trem2*R47H mice in behavioral assays

As part of the comprehensive phenotypic characterization, all mice were evaluated through a behavioral testing pipeline consisting of open field, spontaneous alternation ( $y$-maze), rotarod motor coordination, and running wheel activity assessments (Supplemental Tables 2-5). Age-dependent impairments were observed across all genotypes from 4 to 24 months of age in locomotor activity as measured by open field and entries in the y-maze, motor coordination as measured by rotarod, and wheel running activity (Figure 2; Supplemental Figure 2; Supplemental Figure 4). Spatial working memory was preserved up to 24 months of age with no deficits observed across genotype relative to 4 months of age. Interestingly, in the rotarod motor coordination assay, there was a greater impairment observed in B6. Trem2* $R 47 H$ relative to B6.APOE4.Trem2* $R 47 H$ or B6.APOE4 alone at 24 months (Figure $2 A, B$ ).

12 Compared to 4-month-old animals, we observed a decrease in activity during the active period (dark phase)

13 by 24 months (Supplemental Figure 4). At 24 months of age, home cage running wheel activity suggested

14 a correlation between increased activity during the active period and expression of the APOE 4 allele (both

15 B6.APOE4 and B6J.APOE4/Trem2* R47H mice) by way of total distance traveled and day-time activity in

16 B6.Trem ${ }^{*} R 47 H$ mice (Supplemental Figure 4 E,F). In addition to monitoring physical wellness and

17 behavior of otherwise healthy mice, we also wanted to track the effects of sex and genotype on animal 18 longevity, in the absence of amyloid-associated AD. For this analysis, mortality was defined as subjects

19 that were found dead with no obvious signs of infection, trauma, or intervention during daily monitoring.

20 Mortality risk for each allele was determined by comparing survival scores of cohorts aged to 24 months.

21 Overall, females had a greater risk than males, and survival probabilities were lowest in both sexes for

22 animals expressing both LOAD risk alleles (Supplemental Figure 5).

\section{PET/MRI identified age- and genotype-dependent differences in glycolysis and tissue perfusion}

In an effort to understand the role of risk alleles on regional glycolysis and tissue perfusion, translationally relevant regional measures were acquired via 18F-FDG and 64Cu-PTSM PET/MRI and

27 autoradiography, respectively. By 12 months glycolysis was altered in key brain regions associated with 28 sensory integration, cognition, vision and motor function in B6.APOE4 and B6.APOE4.Trem2*R47H mice, 
1 when compared with controls (Figure 3), and were confirmed via post-mortem autoradiography, which has

2 a 40 folder greater resolution than PET. As expected, these changes were greater in number of regions

3 and magnitude of change in female mice when compared to males (Figure $3 \mathbf{B}, \mathbf{C}$ ). These changes were

4 similarly observed through time, where female mice showed significantly altered glycolysis at 4, 8 and 12

5 months, while male mice largely showed a hypoglycolytic phenotype at 8 months, that was virtually

6 mitigated by 12 months (Supplemental Figure 6). Since these risk alleles can alter metabolic functionality

7 and neuroinflammatory-driven tissue perfusion in an independent manner, we quantitatively measured

8 changes in regional tissue perfusion via 64CU-PTSM PET/MRI and confirmed this via autoradiography.

9 Brain perfusion was significantly lower in regions associated with sensory integration, cognition, vision and

10 motor function in both sexes by 12 months and confirmed via post-mortem autoradiography (Figure 4).

11 Interestingly, these changes were manifested temporally, with the greatest reductions occurring across

12 genotypes and regions in both sexes at 4 months (Supplemental Figure 7). Unlike glycolysis, these

13 changes were largely resolved by 8 months in female mice, while males continued to show regional

14 reduction in perfusion at this same age.

16 Biochemical and neuropathological effects of $A P O E \varepsilon 4$ and $T r e m 2 * R 47 H$ alleles

17 Confirmation of protein expression levels in brain tissue were confirmed for alleles encoding human

18 APOE4 and mouse TREM2 carrying the R47H mutation (Supplemental Figure 8). Similar to reports of

$19 \mathrm{R} 47 \mathrm{H}$ variant-mediated reduction in Trem2 transcript levels (6), TREM2 protein levels in the brains of these

20 animals were also decreased. However, instead of a near knock-out of all TREM2 that has been reported

21 previously (6), levels fell by approximately $50 \%$ in $T r e m 2 * R 47 H$ animals compared to C57BL/6J

22 (Supplemental Figure $8 \boldsymbol{A}, \boldsymbol{B})$. We have previously shown APOE4 protein levels are similar to endogenous

23 mouse APOE (14) and expression of APOE4 appeared similar between male and female

24 APOE4.Trem2* $247 \mathrm{H}$ mice (Supplemental Figure $8 \mathrm{C}$ ) . Additional molecular characterization of these

25 animals showed both age- and genotype-driven differences in levels of cytokines present in the brain and

26 blood (Supplemental Figure 9). IL-6 and KC/GRO concentrations were highest in

27 B6.APOE4.Trem ${ }^{*} R 47 \mathrm{H}$ brain tissue at 8 months, while blood plasma concentrations continued to increase 
with age in those mice (Supplemental Figure $9 B, D, E)$. In multiple occasions a trend appeared to suggest increased cytokine concentrations in mice expressing mutated allele Trem2.

Neuropathological features of AD were then investigated by hematoxylin and eosin (H\&E, structure) and luxol fast blue/cresyl violet (LFB/CV, myelin) staining but did not reveal any gross anatomical changes to tissue architecture or myelin (Figure 5A). Brain sections were also imaged via immunofluorescence and included neuritic plaque-reactive-microglia (X34/Lamp1/lba1), vascular leakage (CD31/lba1/Fibrin), and ThioflavinS (amyloid plaques and neurofibrillary tau tangles) (Figure 5B). No gross abnormalities in cell counts, nor additional neuropathological features were observed in 24 month B6.APOE4.Trem2*R47H mice. We focused particularly on the cortex and hippocampus where episodic memory (hippocampus) and memory behavior (cortex) are regulated. Amyloid plaques and hyperphosphorylated Tau were not observed

11 in mice of any genotype at any age (Figure $5 B$ ).

13 Transcriptional profiling revealed Individual and synergistic effects of APOE\&4, Trem2*R47H, and 14 age

Brain hemispheres from 4 and 24 month male and female B6.APOE4.Trem ${ }^{*} R 47 \mathrm{H}$ mice and single

16 genotype and C57BL/6J controls were assessed using RNA-seq (see Methods). Transcriptomic analysis 17 measured the expression levels (log-transformed TPM counts) of mouse Apoe, Trem2, and human APOE 18 genes across all mouse models (Figure 6 A-C). We observed higher expression of human APOE gene in 19 mice carrying humanized $A P O E \& 4$ (B6.APOE4 and B6.APOE4.Trem2*R47H mice), whereas mouse Apoe 20 gene was highly expressed in $\mathrm{B} 6$ and $T r e m 2^{*} R 47 \mathrm{H}$ mice (Figure $\left.6 \mathbf{A}, \boldsymbol{B}\right)$. As expected based on protein 21 levels (Supplemental Figure 7 A,B), expression of Trem2 was significantly reduced $(p<0.05)$ in

22 B6.Trem2* $R 47 H$ and B6.APOE4.Trem2* $R 47 H$ compared to age-matched B6 (Figure 6C), an effect likely 23 caused by a novel effector splice site and truncation introduced by the R47H mutation. Furthermore, 24 expression level of Trem2 increased with age across all mouse models, but no such patterns were observed 25 in the expression levels of mouse Apoe and human APOE genes (Figure 6 A,B). In addition, there was 26 lower expression of Trem2 in B6.APOE4.Trem2 ${ }^{*} R 47 H$ compared to B6.Trem2 ${ }^{*} R 47 H$ mice at advanced 27 age (24 months), suggesting expression of Trem2 might be suppressed by APOE $\varepsilon 4$. Next, principal 28 component analysis (PCA) identified two distinct clusters corresponding to male and female samples 
separated along the first principal component ( $26 \%$ of total variance), suggesting sex-specific differences are profound in mice (Figure 6D). Analysis of samples from different age groups revealed a gradient of discrimination along the second principal component (14\% of total variance) (Figure 6D2, implying the presence of age-dependent molecular changes in the brain transcriptomes.

To identify molecular effects of the LOAD risk genes, we performed pairwise differential analysis between each genotype (B6.APOE4, B6.Trem2* $R 47 H$ or B6.APOE4.Trem $2^{*} R 47 H$ ) and age- and sexmatched B6 controls. At an early age (4 months), only a few genes were differentially expressed (DEG) ( $p$ $<0.05$ ) for all genotypes for both sexes (Supplemental Figure 10A) and no KEGG pathways were enriched. At 8 months of age, there were 32 DEGs (3 upregulated, 29 downregulated) $(p<0.05)$ in male B6.Trem2* $R 47 H$ mice, and 11 DEGs (2 upregulated, 9 downregulated) $(p<0.05$ ) in female B6.Trem ${ }^{*} R 47 H$ mice (Supplemental Figure 10A). KEGG Pathway analysis identified enrichment of

12 genes involved in immune related pathways such as 'complement and coagulation cascades' and 13 'staphylococcus aureus infection' in the downregulated DEGs in female B6.Trem2*R47H mice (Figure 7).

14 In 8 months old B6.APOE4 mice, a total of 145 genes were significantly differentially expressed (42 15 upregulated, 103 downregulated) $(p<0.05)$ in male mice, whereas a total of 25 genes were differentially 16 expressed (11 upregulated, 14 downregulated) $(\mathrm{p}<0.05)$ in female mice (Supplemental Figure 10A). 17 Pathway enrichment analysis of upregulated genes in male B6.APOE4 mice identified enrichment of 18 'platelet activation' pathway, whereas downregulated genes in female B6.APOE4 mice were enriched for 19 'protein processing in endoplasmic reticulum' pathway (Figure 7). No KEGG pathways were enriched for 20 downregulated DEGs in male B6.APOE4 mice and upregulated DEGs in female B6.APOE4 mice. DEGs in 21 male B6.APOE4.Trem2* $R 47 H$ (7 upregulated, 32 downregulated), and female B6.APOE4.Trem2*R47H 22 mice (2 upregulated, 1 downregulated) $(\mathrm{p}<0.05)$ (Supplemental Figure 10; Figure 7) were not enriched 23 in any KEGG pathway.

At 12 months of age, there were a total of 206 DEGs (118 upregulated, 88 downregulated) in male

25 B6.Trem2* $R 47 H$ mice and 285 DEGs (113 upregulated, 172 downregulated) $(p<0.05)$ in female 26 B6.Trem2* $R 47 H$ mice. The upregulated DEGs in male Trem2* $R 47 H$ mice were enriched in 'RNA transport' 27 and 'spliceosome' pathways (Figure 7; Supplemental Figure 10), while genes in the 'oxidative 28 phosphorylation' pathway were downregulated in male B6.Trem2* $R 47 \mathrm{H}$ mice (Figure 7; Supplemental 
1 Figure 10). Upregulated and downregulated DEGs in female B6.Trem2* $R 47 \mathrm{H}$ mice, were enriched for

2 'RNA transport' and 'lysosome' pathways respectively (Figure 7; Supplemental Figure 10). There were

3 very few DEGs in B6.APOE4 (1 in male, 3 in female) and B6.APOE4.Trem2* $R 47 H$ mice (2 in male, 5 in

4 female) (Supplemental Figure 10) at this age and therefore no enrichment of KEGG Pathways.

At 24 months old, there were a total of 144 DEGs (15 upregulated, 129 downregulated) in the male

6 B6.Trem2 ${ }^{*} R 47 H$ mice and 748 DEGs (359 upregulated, 389 downregulated) $(p<0.05)$ in the female

7 B6. Trem2* $R 47 H$ mice. At this age, B6.APOE4.Trem2* $R 47 H$ mice showed a greater number of DEGs $(p<$ 8 0.05) in both male (24 upregulated, 197 downregulated) and female mice (83 upregulated, 400

9 downregulated) compared to at younger ages (4-12 months) (Supplemental Figure 10), suggesting that

10 the most dramatic transcriptional changes arise between 12 and 24 months. We also found substantial 11 overlap in downregulated DEGs between B6.Trem2* $R 47 H$ and B6.APOE4.Trem2* $R 47 H$ mice for both

12 sexes (Supplemental Figure 10). This suggests that the Trem2* $R 47 \mathrm{H}$ allele is the major driving force in 13 age-dependent transcriptional changes in B6.APOE4.Trem2*R47H mice. Downregulated DEGs were 14 enriched in multiple AD-related pathways such as 'lysosome', 'osteoclast differentiation', 'phagosome", 15 'antigen processing and presentation', cytokine-cytokine receptor interaction', and 'complement and 16 coagulation cascades' in both 24 months old B6.Trem2* $R 47 H$ and B6.APOE4.Trem2 ${ }^{*} R 47 H$ mice $\underline{\text { (Figure }}$

17 7; Supplemental Figure 10). In B6.APOE4 mice, we observed only two DEGs (1 upregulated, 1 18 downregulated) in male and 24 DEGs (1 upregulated, 23 downregulated) in female mice. Downregulated 19 genes in the B6.APOE4 female mice were enriched in 'NOD-like receptor signaling' pathway (Figure 7;

20 Supplemental Figure 10). We did not observe any enriched KEGG pathways in the upregulated list of 21 genes across all genotypes at 24 months.

23 Home cage voluntary wheel running increased oxidative phosphorylation pathway in 24 APOE4.Trem2*R47H mouse brains

25 In the human population, sedentary lifestyle is correlated with an increased risk of LOAD (45-47).

26 Therefore, to determine if physical activity influenced transcriptional changes in B6.APOE4.Trem2* $R 47 H$

27 mice, a running wheel was provided in the home cage of 22 month old male mice for two months. Brain

28 tissue from these animals was profiled by RNA-seq. A total of 292 DEGs (108 upregulated, 184 
downregulated) were identified in the running B6.APOE4.Trem2* $R 47 \mathrm{H}$ mice compared to 24 months old

2 B6 male mice. Enrichment analysis identified multiple enriched KEGG pathways such as "oxidative

3 phosphorylation", "thermogenesis", and "retrograde endocannabinoid signaling" in the upregulated list of

4 genes, whereas immune system associated pathways were enriched in the downregulated list of genes

5 (Supplemental Figure 11A). To identify the effect of exercise, running B6.APOE4.Trem2*R47H male mice

6 were compared with sedentary 24 month old B6.APOE4.Trem $2^{*} R 47 \mathrm{H}$ male mice and there was a total of

7600 DEGs (312 upregulated, and 288 downregulated). Upregulated DEGs were enriched in pathways such

8 as "oxidative phosphorylation" and "Ribosome" (Supplemental Figure 11A). The expression of these

9 upregulated DEGs enriched for oxidative phosphorylation showed reduced expression in age- and sex-

10 matched B6.APOE4 and B6.Trem2*R47H compared to running B6.APOE4.Trem2*R47H mice

11 (Supplemental Figure 11B). Finally, the expression of the upregulated DEGs associated with oxidative

12 phosphorylation pathway were assessed in transcriptional data from AMP-AD. Reduced expression of

13 these running signature genes was observed in AD cases compared to controls across multiple brain

14 regions such as parahippocampal gyrus (PHG) and frontal pole brain regions (FP) (Supplemental Figure

$15 \underline{11 C}$ ). This suggests that exercise induces beneficial effects on health by increasing the expression of

16 oxidative phosphorylation pathway genes that are down regulated across multiple brain regions in $A D$

17 patients.

19 DISCUSSION

20 MODEL-AD was established in response to the many shortcomings of existing mouse models of

21 AD. Aspects of human pathology have been replicated in mouse strains, most prominently the formation of

22 beta amyloid plaques via transgenic over-expression of brain-specific mutant human amyloid beta

23 precursor protein $(A P P)$, presenilin-1 (PS1), and/or microtubule associated protein tau (MAPT) bearing

24 familial Alzheimer's disease (FAD) mutations (48). Legacy preclinical models rely heavily on alleles that

25 overexpress transgenes, resulting in the removing or masking of important human-relevant biological

26 interactions. These mouse strains have been invaluable for understanding the molecular and behavioral

27 phenotypes of early-onset Alzheimer's disease (EOAD) driven by rapid and robust formation of plaques

28 and tangles in the brain and correlating hyperactivity which is a confound of many cognitive behaviors. 
However, LOAD is $\sim 20 \mathrm{x}$ more prevalent than EOAD and further implicates aging, inflammation, environmental, and many more genetic risk factors in disease development. Our APOE4.Trem ${ }^{*} R 47 H$ mice were healthy late into life allowing a better understanding of the effect of $A D$ risk factors in the context of aging (Supplemental Figure 5). As the heterogeneity of this disease becomes more appreciated, so is the importance of appropriate disease staging. Molecular targets of interest may only be available during particular evolving disease stages: debris (cell fragments, plaques, tangles, etc.) accumulates over time and inflammation, interruption/loss of neuronal function all also change with disease progression. In light of the repeated short-comings of "fit-for-all" therapies, efforts may be better directed at targeted therapies (19, 49, 50). Faithfully modeling a complex, polygenic disease will be aided by the creation of platform strains that carry multiple genetic risk factors to motivate with a scientific rationale rather than a grant-focused one.

11 Of the candidate risk variants identified, expression of the $\varepsilon 4$ allele of $A P O E$ and the R47H mutation in

12 TREM2 were identified as the strongest candidates for initial development of a novel LOAD mouse strain.

13 Introduction of the $\mathrm{R} 47 \mathrm{H}$ mutation into Trem2 resulted in the creation of a novel murine splice site yielding

14 a decrease in approximately $50 \%$ of TREM2 protein (Supplemental Figure 8 A, B), and 20\% decrease in

15 Trem2 transcript (Figure $\mathbf{6 C}$ ). In the absence of amyloid plaque insult, the effects on microglia response

16 due to the mutation and decreased expression are difficult to decipher. Similar models have shown similar

17 decreases in R47H-mediated Trem2 expression and function (51-53). Efforts are ongoing to develop a

18 Trem2 allele expressing the full-length $\mathrm{R} 47 \mathrm{H}$ risk factor at levels similar to wild-type Trem2. $A P O E_{\varepsilon} 4$ is 19 strongly associated with disease development and severity $(15,54)$ and at least one allele is present in 20 approximately $65 \%$ of $A D$ patients (55). Unfortunately, endogenous Apoe in mice does not express the 21 isoform diversity seen from the $A P O E$ allele in humans. Insertion of humanized $A P O E$ alleles into mouse 22 genomes has been a successful strategy to dissect the biology of APOE isoforms in mice $(17-20,56)$. The

23 MODEL-AD APOE $\varepsilon 3$ and $A P O E \varepsilon 4$ allelic series on a C57BL/6J background has subsequently be shown to 24 consistently reproduce the biology presented in other APOE mouse models and most importantly, human 25 patients $(14,56)$. Therefore, APOE4 formed the basis for multiple platform strains that include: 26 B6.APOE4.Trem2*R47H (for which we have provided the identifier 'LOAD1').

27 APOE binds to high-density lipoproteins to facilitate cholesterol and phospholipid transport to LDL 28 receptors. As expected, $(16,56)$, mice expressing the humanized $A P O E \varepsilon 4$ allele showed decreased plasma 
lipoprotein levels across all time points (Supplemental Table 2). We observed an age-dependent decrease

2 in glucose levels across both sexes and all genotypes (Figure 1L), an indication of increased frailty and

3 aging that is common in aging-related-disease studies (57). The contributions of metabolic and vascular

4 factors are strongly implicated in disease progression, and how APOE or any other metabolic trait, via

5 systemic pathways, can influence CNS function should be a continued focus for intervention (58). The brain

6 has one of the richest networks of blood vessels and are especially vulnerable (block or reduce blood flow,

7 oxygen and nutrients). For example, the respective influence of $A P O E \varepsilon 4$, with and without $T r e m 2{ }^{*} R 47 H$

8 expression, in regional changes in brain glycolytic metabolism (59) (Figure 3; Supplemental Figure 6),

9 tissue perfusion $(60,61)$ (Figure 4; Supplemental Figure 7) observed in human AD remain under further

10 study. Additional analyses of this APOE4 allele have also revealed changes in cholesterol metabolism and

11 transcriptional signatures in the brain compared to carriers of the APOE3 allele (14).

12 Despite the heterogeneity of $A D$, age is the strongest risk factor in the human population. As an

13 aging disease, monitoring and evaluating mouse models in relation to age is crucial for understanding onset

14 and progression of disease over time. We selected timepoints that reflected different life stages of an adult

15 mouse: Mature, by 3-6 months (beyond development but not yet affected by senescence); Middle-aged,

16 10-14 months (some senescent changes detected in some, not all, biomarkers of aging); and Aged, 18-24

17 months (senescent markers can be observed in all animals) (62). Non-invasive testing of motor activity,

18 behavior, and cognition have been shown to be reliable phenotypes for staging disease onset and trajectory

$19(63,64)$. Aged mice equally displayed the expected trial dependent increases in consecutive rotarod trials

20 and decreases in total distance traveled over time in the open field assay, regardless of genotype

21 (Supplemental Figure 3). Animal activity, measured by total distance traveled during open field assays

22 (Figure 2 and Supplemental Figure 2), also decreased equally with age. Similarly, home cage wheel

23 running assays, which provide a more comprehensive activity phenotype than the 1-hour open field test,

24 also showed a decrease in activity levels during the active (dark phase) (63) (Supplemental Figure 4).

25 Interestingly, at 24 months some indications of increased activity during the active phase in animals carrying

26 the $A P O E \varepsilon 4$ allele (both B6.APOE4 and B6J.APOE4/Trem2*R47H) and day-time activity in only

27 B6.Trem2* $R 47 H$ mice suggesting a dominant phenotype produced by expression of $A P O E \varepsilon 4$

28 (Supplemental Figure 4 C,D; Supplemental Figure 10). Further consideration of genotype influences in 
behavioral, biometric, and molecular testing by statistical analysis was restricted by breeding strategy. Only littermate animals are considered for statistical comparisons, preventing C57BL6/J and B6.Trem2*R47H animal genotypes from inclusion within timepoints.

In the absence of additional environmental or genetic risk factors, B6.APOE4/Trem $2^{*} R 47 \mathrm{H}$ mice did not display penetrant behavioral phenotypes beyond the expected aging-related changes but did exhibit decreased survival probabilities by 24 months (Supplemental Figure 5). Very few C57BL/6J mice succumbed during the 24-month aging process $(<5 \%)$, whereas mortality was higher in mice expressing both LOAD alleles in both males ( 20\%) and females ( 35\%). Male mice expressing either allele alone had survival probabilities similar to C57BL/6J, whereas females with $A P O E \varepsilon 4$ or $T r e m 2^{*} R 47 H$ showed a mortality rate of $\sim 20 \%$. Therefore, it would seem that these two LOAD risk alleles show an equal and

11 additive risk when expressed together, but in females their interaction appears synergistic.

We investigated the molecular signatures in the brain transcriptomes of LOAD mouse models at

13 different ages in both sexes. We identified age-dependent molecular changes associated with LOAD

14 pathologies in mouse models. Introduction of the $\mathrm{R} 47 \mathrm{H}$ mutation revealed a novel Trem2 isoform identical 15 to primary transcript, but truncated by 119 bp from its start position in exon 2 (See Materials and Methods)

16 (44). Expression of this novel isoform resulted in a decrease in both transcript and protein compared to 17 wild-type Trem2 carriers (Figure 6C; Supplemental Figure 8 A,B). Despite the decrease in Trem2 expression, mouse models carrying $\mathrm{R} 47 \mathrm{H}$ mutation in Trem2 gene did not exhibit any significant

19 transcriptional changes at young age, in contrast APOE4 mice exhibited significant changes only at 8 20 months of age. We further identified significant downregulation of genes associated with oxidative 21 phosphorylation pathway in the 12 months old B6.Trem2* $R 47 \mathrm{H}$ mice, suggesting that the oxidative 22 phosphorylation could be prominent early feature for the onset of neurodegeneration/inflammation process.

23 Subsequently, multiple immune related processes were disrupted in 24 months old $B 6$. Trem2 ${ }^{*} R 47 H$ and 24 B6.APOE4.Trem2*R47H mice, supporting the profound relationship between aging, Trem2 and AD. 25 Interestingly, at 12 months of age we did not observe any significant transcriptional changes in 26 B6.APOE4.Trem2*R47H mice compared to control mice, suggesting that the effect of Trem2 gene is 27 suppressed due to the presence of $A P O E \& 4$. Similarly, when mouse models were compared with human 28 co-expression modules, we observed strong negative correlation between the B6.Trem $2^{*} R 47 \mathrm{H}$ mice and 
1 immune-related human co-expression modules from multiple brain regions, and this inflammatory response

2 is dampened in the presence of $A P O E_{\varepsilon} 4$ in the B6.APOE4.Trem2*R47H mice. Distinct mouse models

3 showed concordance with distinct human co-expression modules reflecting a different transcriptional

4 response driven by the human $A P O E_{\varepsilon} 4$ and $T r e m 2^{*} R 47 H$ risk variants. We also observed age dependent

5 shift in co-expression patterns associated with LOAD pathologies. A strong negative correlation between

6 co-expression modules associated with cell cycle and DNA repair was observed in the early-aged mouse

7 B6.APOE4 model, whereas advanced-aged B6.APOE4 female mice showed strong positive correlation

8 with these co-expression modules. This overlap with human late-onset co-expression signatures early in

9 life was observed for a number of different brain regions and was absent in Trem2*R47H knock-in mice.

10 Furthermore, aged B6.Trem2* $R 47 \mathrm{H}$ mice showed a moderate overlap with several human neuronal co-

11 expression modules enriched for genes that play an important role in synaptic signaling and myelination.

12 At advanced age, a strong correlation between the mouse models and immune related human co-

13 expression modules highlights the important role of the LOAD associated APOE\&4 and TREM2 R47H

14 variant in Alzheimer's related immune processes. Our experiments predict that $A P O E \varepsilon 4$ functions through

15 the suppression of effects brought out by expression of the $T r e m 2{ }^{*} R 47 H$ allele: 455 genes are upregulated

16 by $T R E M 2^{R 47 H}$ but suppressed by $A P O E \varepsilon 4$ for (Supplemental Figure 10). Our results mirror some

17 emerging evidence that $A P O E \mathcal{E} 4$ suppresses $T r e m 2^{*} R 47 H$ in $A D$ risk, that there are some suggestions that

$18 A P O E \& 4$ carriers don't have increased AD risk with $T r e m 2^{*} R 47 H$ and $T r e m 2^{*} R 47 H$ only increases risk on

$19 A P O E \& 3$ carriers $(65,66)$. Additionally, we observe more differentially expressed genes at middle age than

20 at a later age supporting evidence of an earlier aging phenotype than C57BL/6J mice, with a realignment

21 of transcriptomes at later timepoints $(58,67)$. We employed a weighted gene co-expression network

22 analysis (WGCNA) used to identify modules of correlated genes. Each module was tested for differential

23 expression by strain, then compared with human postmortem brain modules from the Accelerating

24 Medicine's Partnership for AD (AMP-AD) to determine the LOAD-related processes affected by each

25 genetic risk factor $(6,68,69)$. This will be a useful tool in identifying differentially expressed genes correlated

26 with molecular pathways tied to inflammation and identifying a mouse strain that exhibits a similar

27 transcriptional signature to human patients with true neuroinflammation. 
Amyloid plaque formation is a primary diagnostic measure of Alzheimer's disease with both APOE and TREM2 linked to amyloid deposition (2, 4, 9, 40, 48, 67, 70, 71). For example, TREM2 can bind amyloid, altering microglial function, linking the TREM2-APOE pathway directly to amyloid-driven disease progression $(72,73)$. Loss of functional Trem2 in mice resulted in plaques that contained reduced amounts of $\mathrm{APOE}$ and promoted amyloidogenesis in mice by reducing microglial function $(71,74)$ indicating that microglia, through TREM2 mediated signaling, can regulate APOE co-deposition around amyloid deposits. Further, TREM2 KO prevented infiltration of blood-derived myeloid cells and ameliorated plaque burden in APP/PS1 mice (40) and disease-related mutations impair many of its functions (75). However, current amyloidogenic mouse models develop amyloid plaques at very young ages, within a few months (Supplemental Figure 7B), whereas in human patients the average age of AD onset is at older ages, $\sim 80$ years, potentially causing the disparity in therapeutic outcomes between mouse models and human

12 patients. In the absence of amyloid deposition, many hallmarks of LOAD can be investigated for APOE\&4-

13 and Trem2-influenced effects that precede and may contribute to onset of AD. However, current work is 14 evaluating the effects of APOE and TREM2 risk alleles in the context of humanized Abeta. For instance, 15 we are currently evaluating a novel B6.APOE4.Trem2* $R 47 H . h A b e t a$ (LOAD2) strain and in the process of 16 incorporating humanized Tau (MAPT) alleles into forthcoming strains. These novel platform or AD17 sensitized strains (e.g., LOAD1, LOAD2 etc.) are being used to assess the contribution of additional genetic 18 risk factors identified through genetic and genome-wide association approaches using LOAD1 as a platform 19 strain. These include variations in genes commonly associated with AD including ABCA7, PLCG2, CR1, 20 BIN1 and SORL1. The new strains are prioritized for extensive phenotyping using a primary screening 21 approach centered on transcriptional profiling of nearly 800 genes known to be differentially expressed in 22 human $A D$ brains compared to unaffected controls. (76). Platform stains are also ideal for studying age23 dependent effects of environmental risk factors, such as diet, as well as genetic context. Deeper analysis of $A P O E 4 . T r e m 2^{*} R 47 H$ transcriptional data, in vivo imaging, and neuropathology samples are continuing

25 and will be detailed in future publications. Amendments to the current phenotyping strategy are also in 26 consideration to expand characterizations of the metabolome, proteome, and electrophysiology of LOAD 27 animals. In subsequent studies utilizing new mouse strains, the utility of the APOE4.Trem2*R47H datasets 28 will grow. Ultimately, strains carrying combinations of risk factors that more closely align with human 
disease will be incorporated into the pre-clinical testing core of MODEL-AD to assess the potential of

2 prioritized compounds to treat AD.

\section{METHODS}

6 Model backgrounds

7 All animals were obtained from The Jackson Laboratory. Mouse models of Late-onset Alzheimer disease 8 (LOAD) developed by MODEL-AD (Model Organism Development \& Evaluation for Late-onset Alzheimer's

9 Disease) are congenic to the C57BL/6J (JAX\# 000664) (B6) strain. Genetic variants, identified from human

10 data compiled by the AMP-AD (Accelerating Medicines Partnership - Alzheimer's Disease) project, 11 expressed in MODEL-AD-generated strains are listed on the MODEL-AD strain table at https://www.model-

12 ad.org/strain-table/, along with relevant links for allele descriptions, data, distribution, and legal disclaimers.

14 B6J.APOE4.Trem2*R47H (LOAD1) mice

15 The B6.APOE4.Trem2*R47H (LOAD1) double mutant strain created at The Jackson Laboratory in Bar

16 Harbor, Maine carries two primary risk alleles found in Alzheimer's disease patients. The humanized ApoE

17 knock-in allele, in which a portion of the mouse Apoe gene (exons 2, 3, a majority of exon 4, and some 3'

18 UTR sequence) of the mouse Apoe gene was replaced by the corresponding sequence of the

19 human APOE4 gene (available as B6(SJL)-Apoe ${ }^{\mathrm{tm} 1.1(\mathrm{APOE} * 4) A d i u j / J}$; https://www.jax.org/strain/027894) (14).

20 The second allele, Trem2, contains the R47H point mutation and two additional silent mutations (available

21 as C57BL/6J-Trem2em1Adiuj/J; https://www.jax.org/strain/027918). The human R47H variant, when

22 expressed in mouse brains, also confers a novel splice variant due to a cryptic slice acceptor site in exon

232 (52). See additional information in the Jackson Laboratory APOE4.Trem2R47H mouse (JAX strain $24 \#$ \#28709) strain data sheet. APOE4.Trem2R47H mouse strain data sheet at

25 https://www.jax.org/strain/028709.

27 Animal housing conditions 
All experiments were approved by the Animal Care and Use Committee at The Jackson Laboratory and the Institutional Animal Care and Use Committee at Indiana University. To minimize gene expression variation between mice, animal housing conditions were replicated between both Bar Harbor and Indianapolis campuses. Mice were bred in the mouse facility at Indiana University or The Jackson Laboratory and maintained in a 12/12-hour light/dark cycle, consisting of 12 hours-ON 7am-7pm, followed by 12 hoursOFF. Room temperatures are maintained at $65-75^{\circ} \mathrm{C}$ with $40-60 \%$ humidity. All mice were housed in positive, individually ventilated cages (PIV). Standard autoclaved 6\% fat diet, (Purina Lab Diet 5K52) was available to the mice ad lib, as was water with acidity regulated from $\mathrm{pH}$ 2.5-3.0. All breeder and experimental mice were housed in the same mouse room and were aged together. All behavioral characterization was conducted in the Mouse Neurobehavioral Core Facility (MNBF) at The Jackson

11 Laboratory. Briefly, mice were relocated from the housing room in which they were reared to the MNBF in

12 an adjacent building on the Bar Harbor campus. Mice were individually housed at minimum 5 days prior to 13 behavioral testing. The dedicated MNBF housing room consists of PIV caging with temperature controlled 14 at a setting of $72 \pm 2^{\circ} \mathrm{F}$ and humidity at $50 \pm 20 \%$. The testing facility was on a $12: 12 \mathrm{~L}: \mathrm{D}$ schedule (lights on 15 at 6:00am) with all testing performed during the light cycle (typically between 7:00am and 5:00pm, with the 16 exception of wheel running which was continuous 24-hour testing for up to 5 days). All subjects were 17 randomized and counterbalanced for testing order across multiples of instrumentation and time of day for 18 each test day, with a simplified testing ID number (e.g., \#1-100), with all technicians blinded to genotype

19 (e.g., coded as A, B, C, etc.). The blind was maintained throughout testing and until after the data were 20 analyzed with no subjects or data excluded based on any mathematical outliers.

\section{Behavioral testing}

23 Behavioral tests were conducted as previously reported (63) in the following order with at minimum a 1-2-

24 day rest period between tests: Frailty assessment with core body temperature recording, open field test, 25 spontaneous alternation, rotarod, and wheel running activity. On each test day, subjects were transported 26 from the adjacent housing room into the procedure room, tails were labeled with a non-toxic permanent 27 marker with the assigned subject ID number, and subjects were left to acclimate undisturbed to the testing 28 environment for a minimum 60 minutes prior to testing. Between subjects, all testing arenas were sanitized 
1 with $70 \%$ ethanol solution and dried prior to introducing the next subject. Lighting in the testing rooms were consistent with the housing room ( 500 lux) unless where specifically noted. At minimum 5 days post the

3 conclusion of behavioral testing, mice were sent for tissue harvesting.

Frailty assessment

6 Similar to as previously described (53), subjects were individually evaluated for the absence or presence 7 of 26 aging related characteristic traits and scored a $0,0.5$, or 1 (based on presence/absence, and severity) 8 for each assessment by a trained observer, blind to genotype/age, and included the following assessments:

9 alopecia; loss of fur color; dermatitis/skin lesions; loss of whiskers; coat condition; piloerection; cataracts; 10 eye discharge/swelling; microphthalmia; nasal discharge; rectal prolapse; vaginal/uterine/penile; diarrhea; 11 vestibular disturbance; vision loss assessed by visual placing upon subject being lowered to a grid; menace

12 reflex; tail stiffening; impaired gait during free walking; tremor; tumors; distended abdomen; kyphosis; 13 body condition; breathing rate/depth; malocclusions; righting reflex. The frailty index score was calculated 14 as the cumulative score of all measures with a maximum score of 26.

\section{Core body temperature}

17 Core body temperature was recorded just prior to the conclusion of the frailty assessment via a glycerol 18 lubricated thermistor rectal probe (Braintree Scientific product\# RET 3; measuring 3/4" L .028 dia. .065 tip)

19 inserted $\sim 2 \mathrm{~cm}$ into the rectum of a manually restrained mouse for approximately 10 seconds. Temperature

20 was recorded to the nearest $0.1^{\circ} \mathrm{C}$ (Braintree Scientific product\#TH5 Thermalert digital thermometer).

\section{Open field activity}

23 Versamax Open Field Arenas $(40 \mathrm{~cm} \times 40 \mathrm{~cm} \times 40 \mathrm{~cm}$; Omnitech Electronics, OH USA) were used for this 24 test. Arenas were housed within sound attenuated chambers with lighting in the testing room and arenas 25 consistent with the housing room ( $\sim 500$ lux $)$. Mice were placed individually into the center of the arena and 26 infrared beams recorded distance traveled $(\mathrm{cm})$, vertical activity, and perimeter/center time. Data were 27 collected in 5-minute time-bins for duration of 60 minutes. 
Spontaneous alternation

2 Mice were acclimated to the testing room under ambient lighting conditions ( $\sim 50$ lux). A clear polycarbonate

$3 y$-maze (in-house fabricated; arm dimensions $33.65 \mathrm{~cm}$ length, $6 \mathrm{~cm}$ width, $15 \mathrm{~cm}$ height) placed on top of

4 an infrared reflecting background (Noldus, The Netherlands), surrounded by a black floor-to ceiling curtain

5 to minimize extramaze visual cues was used for this test. Mice were placed midway of the start arm (A),

6 facing the center of the $y$ for an 8-minute test period and the sequence of entries into each arm are recorded

7 via a ceiling-mounted infrared camera integrated with behavioral tracking software (Noldus Ethovision XT).

8 Percent spontaneous alternation is calculated as the number of triads (entries into each of the three different

9 arms of the maze in a sequence of three without returning to a previously visited arm) relative to the number

10 of alteration opportunities.

\section{Rotarod test for motor coordination}

13 An accelerating Rotarod (Ugo-Basile; model 47600) is used for this test. Lighting in the testing room is 14 consistent with the housing room ( 500 lux). The trial began with mice being placed on the rotating rod (4 $15 \mathrm{rpm}$ ), which accelerates up to $40 \mathrm{rpm}$ over the course of 300 seconds. Each mouse is subjected to 3 16 consecutive trials with an $\sim 1$ min inter-trial interval to allow cleaning of the rod between trials. Latency to 17 fall (sec) is measured. Subjects that fall upon initial placement on the rod, before acceleration begins, are 18 scored as $0 \mathrm{sec}$ for that trial.

Wheel running activity

21 Subjects were individually housed into a clean cage with a running wheel (Med-Associates, Vermont, USA)

22 and with food and water ad libitum. The light cycle was identical to the housing room with 12:12 L:D (lights

23 on at 6:00am). Running Wheels were equipped with a wireless transponder that recorded activity on the

24 running wheels (revolutions) in sync with a computer that time stamps events. Mice were left undisturbed

25 throughout the testing period with the exception of daily welfare checks. Data were evaluated for time spent

26 running ( $\mathrm{min}$ ), total distance traveled (meters), and speed (revolutions per min) over the course of three 24-

27 hour periods 
Behavioral data analysis

2 Prior to data analysis and while still blinded, results were adjusted to exclude data only from mice which

3 could not be tested or which data was not available inclusive of any equipment failures, escape episodes,

4 etc. Subjects were not excluded by any mathematical determination. Data was analyzed under coded

5 genotypes (A, B, C, etc.) within sex, as one-way or two-way ANOVA as appropriate versus sex- and age-

6 matched WT control. The blind was revealed at the conclusion of the data analysis for interpretation.

Fasted blood glucose collection and measurement

9 Fasted mice were placed into a fresh cage, free of food but with fresh water, at 6am - the beginning of the

10 light-ON cycle. Mice were fasted for 6 hours, until 12pm, at which time blood glucose levels were analyzed.

11 Prior to mouse restraint, a Contour Next EZ blood glucose monitor (Ascensia, Parsippany, NJ) was

12 calibrated with Contour glucose control solution and Contour Next test strips. While restraining the animal,

13 with a $5.0 \mathrm{~mm}$ lancet a stab incision was made into and perpendicular to the cheek, located dorsal to the to

14 the cheek skin gland at a distance equal to the height of the eye and caudal distance equal to the length of

15 the eye. One drop of blood, approximately $10 \mu \mathrm{l}$, was applied to a blood glucose test strip and readings were

16 recorded.

18 Fecal collection

19 Parallel with measurement of animal weight, animals were placed in a clean container on a scale. Mouse

20 weight was recorded and upon production, fecal sample was collected with forceps to prevent

21 contamination. Sample was placed in a pre-marked $1.5 \mathrm{~mL}$ tube and snap-frozen immediately on dry ice.

22 Container and forceps were cleaned with $70 \%$ ethanol before collecting from subsequent mice. Fecal 23 samples were stored long term at $-80^{\circ} \mathrm{C}$ until analyzed.

\section{Animal anesthesia}

26 Upon arrival at the terminal endpoint for each aged mouse cohort, individual animals were weighed prior to 27 intraperitoneal administration of either: (A) ketamine (100mg/kg) and xylazine (10mg/kg); or (B) 28 tribromoethanol $(1 \mathrm{mg} / \mathrm{kg})$. Routine confirmation of deep anesthesia was performed every 5 minutes by toe 
1 pinch. First confirming deep anesthetization via toe pinch, an incision along the ventral midline to expose

2 the thorax and abdomen, followed by removal of the lateral borders of the diaphragm and ribcage revealed

3 the heart. If desired, prior to perfusion blood and CSF samples must be collected. To perfuse the animal, a

4 small cut was placed in the right atrium to relieve pressure from the vascular system before perfusing the

5 animal transcardially with 1 XPBS via injection into the left ventricle. Completion of perfusion and clearance

6 of the vascular system was indicated by a blanching of the liver.

Whole Animal Perfusion

9 First confirming deep anesthetization via toe pinch, animals are secured to a surgical board or tray using

10 needles or pins and abdomen wetted with $70 \%$ ethanol followed by an incision along the ventral midline

11 along the entire ventral surface, exposing the underlying muscle of the thorax and abdomen. An additional

12 incision is made into this underlying muscle and cut to puncture the diaphragm, taking care not to cut any

13 major blood vessels or the lungs. To expose the heart the ribcage can be cut along the lateral borders and

14 removed. A small incision is made in the right atrium of the heart to relieve diastolic pressure and begin

15 removal of blood from the vascular system. To clear the vascular system of all blood a butterfly catheter

16 needle is inserted into the left ventricle attached to a perfusion pump. Approximately $10 \mathrm{~mL}$ of $1 \mathrm{xPBS}$

17 solution will clear the system of a $20 \mathrm{~g}$ animal. Once the system has been cleared of blood the liver will

18 appear very pale and PBS will be noticed exiting the right atrium. At this time organs of interest were

19 collected as indicated.

$21 \quad$ Non-fasted blood collection and analysis

22 Blood was collected by cardiac puncture from non-fasted, anesthetized animals (see Perfusion method) at 23 harvest prior to incision of the right atrium and subsequent perfusion. A 25-gauge EDTA-coated needle, 24 attached to a $1 \mathrm{~mL}$ syringe, is inserted into the right atrium of the exposed heart and the plunger gently 25 pulled to slowly aspirate approximately $500 \mathrm{~mL}$ of blood, avoiding entrapping air in the syringe to prevent 26 hemolysis. After removal of the needle from the syringe, the blood was slowly injected into a $1.5 \mathrm{~mL}$ EDTA 27 coated MAP-K2 blood microtainer (363706, BD, San Jose, CA) on ice. Blood tubes were spun at $4^{\circ} \mathrm{C}$ and $284,388 \times \mathrm{xg}$ for 15 minutes. Blood serum is then removed and aliquoted equally into three replicate $1.5 \mathrm{~mL}$ 
1 tubes on ice. Tubes were then snap frozen on dry ice and stored long-term at $-80^{\circ} \mathrm{C}$. Thawed blood plasma 2 collected from non-fasted mice was then analyzed by Beckman Coulter AU680 chemistry analyzer

3 (Beckman Coulter, Brea, CA) and Siemens Advia 120 (Germany) for levels of non-fasted glucose, total 4 cholesterol, LDL (low-density lipoproteins), HDL (high-density lipoproteins), triglycerides, and NEFA (non5 essential fatty acids).

Brain harvest

8 Anesthetized and subsequently perfused animals were decapitated, and heads submerged quickly in cold

9 1xPBS. The skin was cut from the base of the neck, over the top of the skull, between the ears, stopping 10 between the eyes, and separated to either side to expose the skull. The skull was cut dorsally until the 11 cerebellum. Two opposite, horizontal cuts were made in the skull under, but without cutting, the cerebellum 12 to ease separation of the skull at the midline. On the top of the skull, scissor blades were inserted 13 superficially at the bregma and slowly expanded to separate the skull down the midline. With a pair of blunt14 end forceps, the two skull plates were removed to expose the brain. The brain was carefully removed from 15 the skull, weighed, and divided midsagitally, into left and right hemispheres, using a brain matrix. The right 16 hemisphere was quickly homogenized on ice and equally aliquoted into three cryotubes for metabolomic,

17 proteomic, and transcriptomic analysis. Cryotubes were immediately snap frozen on dry ice, and stored 18 long-term at $-80^{\circ} \mathrm{C}$. The left hemisphere was immediately placed in $5 \mathrm{~mL} 4 \%$ PFA at $4^{\circ} \mathrm{C}$ for no less than 24 19 hours, but no longer than 30 hours. The left hemisphere was then moved from PFA solution to $10 \mathrm{~mL} 15 \%$ 20 sucrose at $4^{\circ} \mathrm{C}$ for 24 hours, or until it sinks in the sucrose, when it was then transferred to a $30 \%$ sucrose 21 for 24 hours at $4^{\circ} \mathrm{C}$, or until it sinks in the solution. The left hemisphere was then removed from $30 \%$ sucrose 22 solution, snap frozen on a flat mold, cut-side down, floating in 2-methylbutane solution cooled by dry ice.

23 Once frozen the left hemisphere is then placed into a cryotube and stored at $-80^{\circ} \mathrm{C}$ until used for microtome 24 sectioning and immunohistochemistry analysis. Immunohistochemistry and microscopy imaging

27 During harvest, whole mouse brains were removed and weighed. Using a brain matrix, left and right 28 hemispheres were separated along the midsagittal plane. The left hemisphere was placed in $5 \mathrm{~mL}$ of $4 \%$ 
PFA at $4^{\circ} \mathrm{C}$ overnight, then moved to $10 \mathrm{~mL}$ of $15 \%$ sucrose at $4^{\circ} \mathrm{C}$ overnight, before finally being incubated in $10 \mathrm{~mL}$ of $30 \%$ sucrose at $4^{\circ} \mathrm{C}$ overnight or until brain sinks to bottom of the tube. The left hemisphere was then snap frozen and stored at $-80^{\circ} \mathrm{C}$ until sectioned. Left hemispheres (see preparation in Brain harvest method) were cut via Thermo Scientific HM430 sliding microtome at $25 \mu \mathrm{m}$ thickness. Coronal brain tissue sections were oriented to capture the cortex and hippocampus at approximately Bregma: $-2.75 \mathrm{~mm}$ and Interaural 1.05mm. Each section was placed into cryoprotectant buffer (37.5\% 1xPBS, 31.25\% glycerol, $31.25 \%$ ethylene glycol) for immediate use or long-term storage at $-20^{\circ} \mathrm{C}$. Each section was tracked so as to store 100 sections equally distributed over 10 groups, so each of the 10 groups had equal representation of the 10 sections from forebrain to hindbrain and hippocampus. Of these 10 groups of 1025 -micron sections, 7 will be used for standardized staining combinations highlighting cell types and markers of interest: (1) Vascular [CD31/lba1/Fibrin/DAPI]; (2)

; (2) Neuritic plaques [Lamp1/lba1/X34];

(3) Astrocytes and microglia [GFAP/lba1/S100b/DAPI];

; (4) Neurons [NeuN/Ctip2/DAPI];

(5) Plaques [ThioS];

(6) Luxol Fast

russian blue (Iron stain). Floating sections were then blocked prior to immunohistochemical staining and mounting. After blocking slides with $10 \%$ normal donkey serum or normal goat serum diluted in $1 \mathrm{xPBS}+0.5 \%$ Triton wash buffer all antibodies were washed floating in 1xPBT (1x PBS with $0.5 \%$ Triton) wash buffer after blocking for 1 hour at room temperature on shaker in $10 \%$ NGS (normal goat serum) or 10\% NDS (normal donkey serum) in 1xPBT. Secondary

18 antibodies were incubated in $10 \%$ NGS or NDS in 1XPBT for 1 hour at room temperature, followed by

19 washes in 1 XPBT before mounting on to slides. Each staining combination of 10 sections were placed onto 20 one slide. DAPI, X34, and ThioS stains were performed on the slide following immunostaining. Slides were 21 then imaged on a Leica Versa slide scanner, automated fluorescent microscope system (Leica, Allendale, $22 \mathrm{NJ}$ ). For further analysis, regions of the cortex and hippocampus were processed using CellProfiler 23 (Cambridge, MA) or Imaris (Bitplane, Concord, MA) software to quantify cell counts, fluorescence intensity, 24 and surface area ratios. [CD31 (R\&D Systems, MAB3628, 1:500), Iba1 (Wako, 019-19741, 1:300), Fibrin 25 (abcam, ab118533, 1:500), DAPI (1:1000), Lamp1 (abcam, ab25245, 1:500), X34 (0.04\% in 40\% ethanol), 26 GFAP (Origene, AP31806PU-N, 1:1000), S100b (Thermo Fisher, PA175395, 1:1000), NeuN (abcam, 27 ab104225, 1:500), Ctip2 (abcam, ab18465, 1:1000), and Thios (1\% in 50\% ethanol)] 
Radiopharmaceuticals

2 Regional brain glycolytic metabolism was monitored using 2-[18F]-fluoro-2-deoxy-D-glucose (18F-FDG)

3 and was synthesized, purified, and prepared according to established methods (77), where clinical unit

4 doses ranging from 185 to $370 \mathrm{MBq}$ (5 to $10 \mathrm{mCi}$ ) were purchased from PETNet Indiana (PETNET Solutions

5 Inc). To evaluate region brain perfusion, Copper(II) pyruvaldehyde bis(N4-methylthiosemicarbazone)

6 labeled with 64Cu (64Cu-PTSM) was synthesized, purified, and unit doses (i.e., 370 to $740 \mathrm{MBq}$ (10 to 25

$7 \mathrm{mCi})$ ) dispensed by the PET Radiochemistry Core Facility at Indiana University according methods

8 described previously $(78,79)$.

10 Magnetic Resonance Imaging (MRI)

11 To provide high contrast grey matter images, at least two days prior to PET imaging, mice were induced

12 with $5 \%$ isoflurane (balance medical oxygen), placed on the head coil, and anesthesia maintained with 1-

$133 \%$ isoflurane for scan duration. High resolution T2-weighted (T2W) MRI images were acquired using a 3T

14 Siemens Prisma clinical MRI scanner outfitted with a dedicated 4 channel mouse head coil and bed system

15 (RapidMR, Columbus OH). Images were acquired using a SPACE3D sequence (80) using the following

16 acquisition parameters: TA: 5.5min; TR: 2080ms; TE: 162ms; ETL: 57; FS: On; Ave: 2; Excitation Flip

17 Angle: 150; Norm Filter: On; Restore Magnetization: On; Slice Thickness 0.2mm: Matrix: 171x192; FOV:

$1835 \times 35 \mathrm{~mm}$, yielding $0.18 \times 0.18 \times 0.2 \mathrm{~mm}$ resolution images. At the completion of the imaging period, mice

19 we returned to their warmed home cages, and allowed to recover.

\section{Positron Emission Tomography (PET) Imaging}

22 To evaluate changes in cerebral glycolysis (18F-FDG) and cerebral perfusion (64Cu-PTSM) mice were

23 placed in a restrainer and consciously injected into the peritoneal or tail vein, respectively, with 3.7-11.1

$24 \mathrm{MBq}(0.1-0.3 \mathrm{mCi})$ of purified, sterile radiotracer, where the final volume did not exceed $10 \%$ of the animal's

25 body weight. Each animal was returned to their warmed home cage and allowed 30 min (18F-FDG) or 5

$26 \mathrm{~min}(64 \mathrm{Cu}-\mathrm{PTSM})$ to allow for uptake and cellular trapping $(81,82)$. Post-uptake, mice were induced with

$275 \%$ isoflurane gas, placed on the scanner imaging bed, and anesthesia maintained at $1-3 \%$ isoflurane

28 (balance medical oxygen) during acquisition. In all cases, calibrated PET acquisition was performed in list 
mode for 15 (18F-FDG) or 30 (64Cu-PTSM) min on an IndyPET3 scanner (83), where random prompts did

2 not exceed $10 \%$ of the total prompt rate. Post-acquisition, the images were reconstructed into a single-

3 static image with a minimum field of view of $60 \mathrm{~mm}$ using filtered-back-projection (FBP), and were corrected

4 for decay, random coincidence events, and dead-time loss (84).

6 Autoradiography

7 To provide secondary confirmation of the in vivo PET images, and to quantify tracer uptake regionally, 8 brains were extracted post rapid decapitation, gross sectioned along the midline, slowly frozen on dry ice,

9 then embedded in cryomolds with Optimal Cutting Temperature (OCT) compound (Tissue-Tek). Thin frozen

10 sections $(20$ um) were obtained via cryotomy at prescribed bregma targets $(n=6$ bregma/mouse, 6 11 replicates/bregma) according to stereotactic mouse brain coordinates (85). Sections were mounted on

12 glass slides, air dried, and exposed on BAS Storage Phosphor Screens (SR 2040 E, Cytiva Inc.) for up to 1312 hrs. Post-exposure, screen were imaged via Typhoon FL 7000IP (GE Medical Systems) phosphor14 imager at 25 um resolution along with custom $18 \mathrm{~F}$ or $64 \mathrm{Cu}$ standards described previously (86).

16 Image Analysis

17 All PET and MRI images were co-registered using a ridged-body mutual information-based normalized 18 entropy algorithm (87) with 9 degrees of freedom, and mapped to stereotactic mouse brain coordinates 19 (85) using Analyze 12 (AnalyzeDirect, Stilwell KS). Post-registration, 56 regions bilateral regions were 20 extracted via brain atlas, and averaged to yield 27 unique volumes of interest that map to key cognitive and 21 motor centers that includes: Agranular Insular Cortex; Auditory Cortex; Caudate Putamen, Cerebellum;

22 Cingulate Cortex; Corpus Callosum; Dorsolateral Orbital Cortex; Dorsintermed Entorhinal Cortex; 23 Dysgranular Insular Cortex; Ectorhinal Cortex; Fornix; Frontal Association Cortex; Hippocampus; Lateral 24 Orbital Cortex; Medial Orbital Cortex; Parietal Cortex; Parietal Association Cortex; Perirhinal Cortex; 25 Prelimbic Cortex; Primary Motor Cortex; Primary Somatosensory Cortex; Retrosplenial Dysgranular Cortex; 26 Secondary Motor Cortex; Secondary Somatosensory Cortex; Temporal Association Cortex, Thalamus; 27 Ventral Orbital Cortex; Visual Cortex. For autoradiographic analysis, tracer uptake was quantified on hemi28 coronal sections by manually drawing regions of interest for 17 regions of interest (i.e. Auditory Cortex, 
1 Caudate Putamen, Cerebellum, Cingulate Cortex, Corpus Callosum, Dorso-intermed Entorhinal Cortex, 2 Dysgranular Insular Cortex, Ectorhinal Cortex, Hippocampus, Hypothalamus, Medial Septum, Primary

3 Motor Cortex, Primary Somatosensory Cortex, Retrosplenial Dysgranular Cortex, Temporal Association

4 Cortex, Thalamus, Visual Cortex) on calibrated phosphor screen at bregma $0.38,-1.94$, and $-3.8 \mathrm{~mm}$ using 5 MCID (InterFocus Ltd). To permit dose and brain uptake normalization, Standardized Uptake Value Ratios 6 (SUVR) relative to the cerebellum were computed for PET and autoradiograms for each subject, genotype, 7 and age as follows:

\begin{tabular}{|l|l|}
\hline $\operatorname{SUVR}(s, R, g, a)=\frac{R(s, g, a)}{C(s, g, a)}$ & (1) \\
\hline
\end{tabular}

where, $s, g, a, R$, and $C$ are the subject, genotype, age, region/volume of interest, cerebellum region/volume

9 of interest. In all cases, region/volumes of interest were analyzed for differences with time and genotype 10 using a Two-Way ANOVA (Prism, GraphPad Inc.), where significance was taken at $p<0.05$.

Immunoprecipitation

13 Tissue samples were homogenized in tissue protein extraction reagent (T-PER ThermoScientific)

14 supplemented with protease and phosphatase inhibitors cocktail (Sigma-Aldrich). Protein concentration 15 was measured using bicinchoninic acid (BCA) (Pierce). Immunoprecipitation was performed by incubating 16 a total of $1500 \mathrm{ug}$ of brain protein extract with $1 \mathrm{ug}$ of biotinylated sheep anti-Trem2 antibody (RnD systems 17 BAF1729) overnight at 4C, followed by incubation with streptavidin sepharose beads (CST 3419) for 6 18 hours at 4C, washed 3 times with ice cold PBS with $0.1 \%$ Tween 20 . Protein was eluted in sample loading 19 buffer with $1 \mathrm{mM}$ DTT followed by separation via Western blot (see below).

21 Western immunoblot

22 Snap-frozen right hemispheres were homogenized by hard tissue homogenizer (USA Scientific, Ocala, FL) 23 and lysed in 1mL RIPA buffer (R0278, Sigma, St. Louis, MO) supplemented with protease and phosphatase 24 inhibitor reagents (1861281, Thermo Fisher Scientific, Waltham, MA). Lysates were incubated for 1 hour at $254^{\circ} \mathrm{C}$ before pelleting insoluble proteins by spinning at $4^{\circ} \mathrm{C}, 11,000 x g$ for 15 minutes. Protein concentration 26 was determined by Bradford protein assay (Biorad, Hercules, CA), according to manufacturer's instructions. 27 Samples were mixed with 10x Laemlli buffer (42556.01, Amsbio, Cambridge, MA), boiled for 10 minutes, 
and run on $12 \%$ SDS PAGE gels (456-1044, BioRad) with colorimetric ladder (RPN800E, GE, Boston, MA).

2 Gels were transferred to PVDF membranes for immunoblotting and imaging using an iBlot2 dry blotting

3 system (Thermo Fisher). Membranes were blocked in 5\% non-fat dry milk in 1xPBS+0.1\% Tween20 for 1

4 hour prior to incubating with primary antibodies diluted in 5\% non-fat dry milk in 1xPBS+0.1\% Tween20 for

51 hour at room temperature. Membranes were washed in $1 x P B S+0.1 \%$ Tween20 before incubating with

6 secondary antibodies diluted in 5\% non-fat dry milk in 1xPBS $+0.1 \%$ Tween 20 . HRP-conjugated secondary

7 antibodies targeting primary antibody host $\lg G$ were incubated at 1 hour at room temperature. Membranes

8 were washed in $1 \times$ PBS $+0.1 \%$ Tween20 before digital imaging with SuperSignal West Pico PLUS

9 chemiluminescent substrate (34579, Thermo Fisher). Images for immunoblot were quantified using Image J

$10 \quad 1.8 .0$ version. Proteins of interest were visualized with the following primary antibodies against: ACTIN

11 (Abcam, ab179467), GAPDH (abcam, ab9483), APOE4 (Novus, NBP1-49529), TREM2 (R\&D Systems,

12 MAB1729), and Alpha-tubulin (Sigma-Aldrich, T9026-100UL).

14 Cytokine Panel Assay

15 Hemibrains were homogenized in tissue homogenization buffer containing fresh protease inhibitor cocktail

16 and aliquoted. Supernatant was utilized for the cytokine analysis. Mouse hemibrain samples were assayed

17 in duplicate using the MSD mouse proinflammatory Panel I, a highly sensitive multiplex enzyme-linked

18 immunosorbent assay (ELISA). The panel quantifies 10 cytokines: interferon gamma (IFN-ץ), interleukin

19 (IL)-1 $\beta$, IL-2, IL-4, IL-6, IL-8, IL-10, IL-12p70, IL-13, and tumor necrosis factor $\alpha$ (TNF $\alpha$ ) from a single small

20 sample volume $(25 \mu \mathrm{L})$ using an electrochemiluminescent detection method (MesoScale Discovery,

21 Gaithersburg, MD, USA). The mean intra-assay coefficient for each cytokine was $<8.5 \%$, based on cytokine

22 standards. Any value that was below the lowest limit of detection (LLOD) for the cytokine assay was

23 replaced with $1 / 2$ LLOD of the assay for statistical analysis.

25 RNA-sequencing experimental design

26 RNA-Seq data were obtained from whole left hemisphere brain samples from APOE4 KI mouse, carrying

27 a humanized version of the prominent $A P O E \& 4$ genetic risk factor for LOAD, and the Trem2*R47H mouse, 28 carrying a rare deleterious variant $\mathrm{R} 47 \mathrm{H}$ allele of Trem2 gene. In addition, a mouse model expressing both 
1 human $A P O E \& 4$ and the $T r e m 2^{*} R 47 H$ mutation was used to compare the transcriptional changes in mice

2 carrying both variants to mice carrying only a single risk allele and B6 controls. Whole-brain left

3 hemispheres were collected at 4, 8, 12, and 24 months of age from both sexes.

4 Study population:

\begin{tabular}{|c|c|c|c|c|c|c|c|c|}
\hline \multirow{2}{*}{ Mouse Models } & \multicolumn{2}{|l|}{$4 M$} & \multicolumn{2}{|l|}{$8 M$} & \multicolumn{2}{|l|}{$12 M$} & \multicolumn{2}{|l|}{$24 M$} \\
\hline & Male & Female & Male & Female & Male & Female & Male & Female \\
\hline C57BL/6J & 12 & 12 & 6 & 6 & 6 & 6 & 7 & 6 \\
\hline APOE4 KI & 13 & 12 & 6 & 6 & 4 & 5 & 5 & 6 \\
\hline TREM2*R47H & 12 & 12 & 6 & 6 & 6 & 6 & 6 & 3 \\
\hline APOE4.TREM2*R47H & 10 & 12 & 5 & 6 & 8 & 5 & 7 & 6 \\
\hline
\end{tabular}

5

12 RNA-Sequencing assay library preparation

13 Sequencing libraries were constructed using TruSeq DNA V2 (Illumina, San Diego, CA) sample prep kits 14 and quantified using qPCR (Kapa Biosystems, Wilmington, MA). The mRNA was fragmented, and double15 stranded cDNA was generated by random priming. The ends of the fragmented DNA were converted into 16 phosphorylated blunt ends. An 'A' base was added to the 3' ends. Illumina $($-specific adaptors were ligated 17 to the DNA fragments. Using magnetic bead technology, the ligated fragments were size-selected and then 
1 a final PCR was performed to enrich the adapter-modified DNA fragments, since only the DNA fragments

2 with adaptors at both ends will amplify.

RNA-Sequencing

5 Libraries were pooled and sequenced by the Genome Technologies core facility at The Jackson Laboratory.

6 All samples were sequenced on Illumina HiSeq 4000 using HiSeq 3000/4000 SBS Kit reagents (Illumina),

7 targeting 30 million read pairs per sample. Samples were split across multiple lanes when being run on the

8 Illumina HiSeq, once the data was received the samples were concatenated to have a single file for paired-

9 end analysis.

11 RNA-Sequencing data processing

12 Sequence quality of reads was assessed using FastQC (v0.11.3, Babraham). Low-quality bases were 13 trimmed from sequencing reads using Trimmomatic (v0.33) (88). After trimming, reads of length longer than

1436 bases were retained. The average quality score was greater than 30 at each base position and 15 sequencing depth were in range of $60-80$ million reads. RNA-Seq sequencing reads from all samples were 16 mapped to the mouse genome (version GRCm38.p6) using ultrafast RNA-Seq aligner STAR (v2.5.3) (89).

17 To measure human $A P O E$ gene expression, we created a chimeric mouse genome by concatenating 18 human $A P O E$ gene sequence (human chromosome 19:44905754-44909393) into mouse genome 19 (GRCm38.p6) as a separate chromosome (referred as chromosome 21 in chimeric mouse genome).

20 Subsequently, we added gene annotation of human APOE gene into mouse gene annotation file.

21 Additionally, we have also introduced annotation for novel Trem2 isoform in mouse gene annotation file 22 (GTF file), that is identical to primary transcript, but truncated exon2 by 119 bp from its start position. 23 Afterwards, a STAR index was built for this chimeric mouse genome sequence for alignment, then STAR 24 aligner output coordinate-sorted BAM files for each sample mapped to chimeric mouse genome using this 25 index. Gene expression was quantified in two ways, to enable multiple analytical methods: transcripts per 26 million (TPM) using RSEM (v1.2.31) (90), and raw read counts using HTSeq-count (v0.8.0) (91). 
1 Differential expression in mouse models was assessed using the R Bioconductor package DESeq2

2 (v1.16.1) (92). DESeq2 takes raw read counts obtained from HTSeq-count as input. Genes with the

3 Benjamini-Hochberg corrected $p$-values $<0.05$ were considered as significantly differentially expressed 4 genes.

6 Principal component analysis

7 We analyzed a total of 234 RNA-Seq samples originating from different mouse models at different ages 8 and sex. First, dispersion parameter for each gene was estimated using DESeq2 R package (92).

9 Afterwards, we applied the varianceStabilizingTransformation (vst) function of DESeq2 (92) to the read 10 count data in order to produce a data matrix in which expression levels are homoscedastic. Finally, we 11 extracted the principal components using the plotPCA function of DESeq2 in R.

\section{Functional enrichment analysis}

14 Functional annotations and enrichment analyses were performed using the $\mathrm{R}$ Bioconductor package 15 clusterProfiler (93), with Gene Ontology terms and KEGG pathways enrichment analyses performed using 16 functions enrichGO and enrichKEGG, respectively. The function compareCluster was used to compare 17 enriched functional categories of each gene module. The significance threshold for all enrichment analyses 18 was set to 0.05 using Benjamini-Hochberg adjusted p-values.

20 Human post-mortem brain cohorts and co-expression module identification

21 Whole-transcriptome data for human post-mortem brain tissue was obtained from the Accelerating

22 Medicines Partnership for Alzheimer Disease-(AMP-AD) consortium, which is a multi-cohort effort to 23 harmonize genomics data from human LOAD patients. Harmonized co-expression modules from the AMP24 AD data sets were obtained from the AD Knowledge Portal (DOI: 10.7303/syn11932957.1). The human co25 expression modules derive from three independent LOAD cohorts, including 700 samples from the 26 ROS/MAP cohort, 300 samples from the Mount Sinai Brain bank and 270 samples from the Mayo cohort. 27 A detailed description on post-mortem brain sample collection, tissue and RNA preparation, sequencing, 28 and sample QC has been provided elsewhere $(12,13,94)$. As part of a transcriptome-wide meta-analysis 
1 to decipher the molecular architecture of LOAD, 30 co-expression modules from seven different brain

2 regions across the three cohorts have been recently identified (6). Briefly, Logsdon et al. (6) identified 2,978

3 co-expression modules using multiple techniques across the different regions after adjusting for co-

4 variables and accounting for batch effects (10.7303/syn10309369.1). A total of 660 co-expression modules

5 were selected based on a specific enrichment in LOAD cases when compared to controls

6 (10.7303/syn11914606). Finally, multiple co-expression module algorithms were used to identify a set of

730 aggregate modules that were replicated by the independent methods (6).

9 Mouse-Human correlation analysis

10 First, we performed differential gene expression analysis for each mouse model compared to age and sex-

11 matched B6 control mice using the limma (95) package in R. Afterwards, we computed correlation between

12 changes in expression (log fold change) for all DE genes in a given module with the fold changes for each

13 mouse model (specified by genotype, sex, diet, and age). Correlation coefficients were computed using 14 cor.test function in $\mathrm{R}$ as:

cor.test( $\log F C(h), \log F C(m))$

16 where $\operatorname{LogFC}(\mathrm{h})$ is the log fold change in transcript expression of human AD patients compared to control

17 patients and $\operatorname{LogFC}(m)$ is the log fold change in expression of mouse transcripts compared to control mouse

18 models. LogFC values for human transcripts were obtained via the AD Knowledge Portal

19 (https://www.synapse.org/\#!Synapse:syn11180450).

21 Statistical analysis

22 Statistical analyses was constrained to comparisons between littermate-controlled subjects only

23 (B6.APOE4 v. B6.APOE4.Trem2*R47H). Student's t-test was employed on data sets differing by a single

24 variable (e.g., age, sex, genotype). ANOVA was used in data sets where multiple factors are considered 25 and combined for possible synergistic effects.

27 Availability of data and materials 
1 The LOAD1 data sets are available via the AD Knowledge Portal (https://adknowledgeportal.org). The AD

2 Knowledge Portal is a platform for accessing data, analyses, and tools generated by the Accelerating

3 Medicines Partnership (AMP-AD) Target Discovery Program and other National Institute on Aging (NIA)-

4 supported programs to enable open-science practices and accelerate translational learning. The data,

5 analyses and tools are shared early in the research cycle without a publication embargo on secondary use.

6 Data is available for general research use according to the following requirements for data access and data

7 attribution (https://adknowledgeportal.org/DataAccess/Instructions).

8 For access to content described in this manuscript see: https://doi.org/10.7303/syn23631984

11 REFERENCES

12

131.2020 Alzheimer's disease facts and figures. Alzheimers Dement. 2020.

14 2. Cacace R, Sleegers K, Van Broeckhoven C. Molecular genetics of early-onset Alzheimer's

15 disease revisited. Alzheimers Dement. 2016;12(6):733-48.

16 3. Services USDoHaH. NIA and the National Plan to Address Alzheimer's Disease: USA.gov; 2021

17 [Available from: https://www.nia.nih.gov/about/nia-and-national-plan-address-alzheimers-disease.

18 4. Blennow K, de Leon MJ, Zetterberg H. Alzheimer's disease. Lancet. 2006;368(9533):387-403.

19 5. Sasaguri H, Nilsson P, Hashimoto S, Nagata K, Saito T, De Strooper B, et al. APP mouse models

20 for Alzheimer's disease preclinical studies. EMBO J. 2017;36(17):2473-87.

21 6. Logsdon B, Perumal TM, Swarup V, Wang M, Funk C, Gaiteri C, et al. Meta-analysis of the

22 human brain transcriptome identifies heterogeneity across human AD coexpression modules robust to

23 sample collection and methodological approach. bioRxiv. 2019:510420.

24 7. Oblak AL, Forner S, Territo PR, Sasner M, Carter GW, Howell GR, et al. Model organism

25 development and evaluation for late-onset Alzheimer's disease: MODEL-AD. Alzheimers Dement (N Y).

$262020 ; 6(1): \mathrm{e} 12110$.

27 8. Karch CM, Goate AM. Alzheimer's disease risk genes and mechanisms of disease pathogenesis.

28 Biol Psychiatry. 2015;77(1):43-51. 
19 . Dourlen P, Kilinc D, Malmanche N, Chapuis J, Lambert JC. The new genetic landscape of

2 Alzheimer's disease: from amyloid cascade to genetically driven synaptic failure hypothesis? Acta

3 Neuropathol. 2019;138(2):221-36.

4 10. Bellenguez C, Grenier-Boley B, Lambert JC. Genetics of Alzheimer's disease: where we are, and 5 where we are going. Curr Opin Neurobiol. 2020;61:40-8.

6 11. Andrews SJ, Fulton-Howard B, Goate A. Interpretation of risk loci from genome-wide association 7 studies of Alzheimer's disease. Lancet Neurol. 2020;19(4):326-35.

8 12. Allen M, Carrasquillo MM, Funk C, Heavner BD, Zou F, Younkin CS, et al. Human whole genome

9 genotype and transcriptome data for Alzheimer's and other neurodegenerative diseases. Scientific Data.

$10 \quad 2016 ; 3: 160089$.

11 13. Wang M, Beckmann ND, Roussos P, Wang E, Zhou X, Wang Q, et al. The Mount Sinai cohort of

12 large-scale genomic, transcriptomic and proteomic data in Alzheimer's disease. Scientific data.

13 2018;5:180185-.

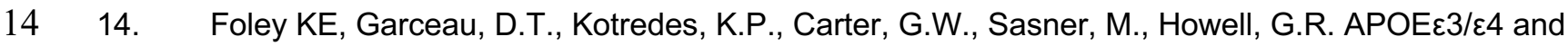

15 APOE $\varepsilon 4 / \varepsilon 4$ genotypes drive unique gene signatures in the cortex of young mice. bioRxiv. 2020;1(1).

16 15. Bu G. Apolipoprotein E and its receptors in Alzheimer's disease: pathways, pathogenesis and

17 therapy. Nat Rev Neurosci. 2009;10(5):333-44.

18 16. Arnold M, Nho K, Kueider-Paisley A, Massaro T, Huynh K, Brauner B, et al. Sex and APOE

19 epsilon4 genotype modify the Alzheimer's disease serum metabolome. Nat Commun. 2020;11(1):1148.

20 17. Lewandowski CT, Maldonado Weng J, LaDu MJ. Alzheimer's disease pathology in APOE

21 transgenic mouse models: The Who, What, When, Where, Why, and How. Neurobiol Dis.

$22 \quad 2020 ; 139: 104811$.

23 18. Balu D, Karstens AJ, Loukenas E, Maldonado Weng J, York JM, Valencia-Olvera AC, et al. The 24 role of APOE in transgenic mouse models of AD. Neurosci Lett. 2019;707:134285.

25 19. Safieh M, Korczyn AD, Michaelson DM. ApoE4: an emerging therapeutic target for Alzheimer's 26 disease. BMC Med. 2019;17(1):64.

27 20. Esquerda-Canals G, Montoliu-Gaya L, Guell-Bosch J, Villegas S. Mouse Models of Alzheimer's

28 Disease. J Alzheimers Dis. 2017;57(4):1171-83. 
1 21. Yamazaki Y, Painter MM, Bu G, Kanekiyo T. Apolipoprotein E as a Therapeutic Target in

2 Alzheimer's Disease: A Review of Basic Research and Clinical Evidence. CNS Drugs. 2016;30(9):773-89.

3 22. Saunders AM, Strittmatter WJ, Schmechel D, George-Hyslop PH, Pericak-Vance MA, Joo SH, et

4 al. Association of apolipoprotein E allele epsilon 4 with late-onset familial and sporadic Alzheimer's

5 disease. Neurology. 1993;43(8):1467-72.

6 23. Strittmatter WJ, Saunders AM, Schmechel D, Pericak-Vance M, Enghild J, Salvesen GS, et al.

7 Apolipoprotein E: high-avidity binding to beta-amyloid and increased frequency of type 4 allele in late-

8 onset familial Alzheimer disease. Proc Natl Acad Sci U S A. 1993;90(5):1977-81.

9 24. Maezawa I, Zaja-Milatovic S, Milatovic D, Stephen C, Sokal I, Maeda N, et al. Apolipoprotein E

10 isoform-dependent dendritic recovery of hippocampal neurons following activation of innate immunity. $\mathrm{J}$

11 Neuroinflammation. 2006;3:21.

12 25. Jeong W, Lee H, Cho S, Seo J. ApoE4-Induced Cholesterol Dysregulation and Its Brain Cell

13 Type-Specific Implications in the Pathogenesis of Alzheimer's Disease. Mol Cells. 2019;42(11):739-46.

14 26. Bell RD, Winkler EA, Singh I, Sagare AP, Deane R, Wu Z, et al. Apolipoprotein E controls

15 cerebrovascular integrity via cyclophilin A. Nature. 2012;485(7399):512-6.

16 27. Methia N, Andre P, Hafezi-Moghadam A, Economopoulos M, Thomas KL, Wagner DD. ApoE

17 deficiency compromises the blood brain barrier especially after injury. Mol Med. 2001;7(12):810-5.

18 28. Maezawa I, Maeda N, Montine TJ, Montine KS. Apolipoprotein E-specific innate immune

19 response in astrocytes from targeted replacement mice. J Neuroinflammation. 2006;3:10.

20 29. Maezawa I, Nivison M, Montine KS, Maeda N, Montine TJ. Neurotoxicity from innate immune

21 response is greatest with targeted replacement of E4 allele of apolipoprotein E gene and is mediated by

22 microglial p38MAPK. FASEB J. 2006;20(6):797-9.

23 30. Chung WS, Verghese PB, Chakraborty C, Joung J, Hyman BT, Ulrich JD, et al. Novel allele-

24 dependent role for APOE in controlling the rate of synapse pruning by astrocytes. Proc Natl Acad Sci U S

25 A. 2016;113(36):10186-91.

26 31. Laskowitz DT, Lee DM, Schmechel D, Staats HF. Altered immune responses in apolipoprotein E-

27 deficient mice. J Lipid Res. 2000;41(4):613-20. 
$1 \quad$ 32. Wang C, Wilson WA, Moore SD, Mace BE, Maeda N, Schmechel DE, et al. Human apoE4-

2 targeted replacement mice display synaptic deficits in the absence of neuropathology. Neurobiol Dis.

$3 \quad 2005 ; 18(2): 390-8$.

4 33. Verghese PB, Castellano JM, Garai K, Wang Y, Jiang H, Shah A, et al. ApoE influences amyloid-

5 beta (Abeta) clearance despite minimal apoE/Abeta association in physiological conditions. Proc Natl

$6 \quad$ Acad Sci U S A. 2013;110(19):E1807-16.

7 34. Shi Y, Yamada K, Liddelow SA, Smith ST, Zhao L, Luo W, et al. ApoE4 markedly exacerbates

8 tau-mediated neurodegeneration in a mouse model of tauopathy. Nature. 2017;549(7673):523-7.

9 35. Ma J, Jiang T, Tan L, Yu JT. TYROBP in Alzheimer's disease. Mol Neurobiol. 2015;51(2):820-6.

10 36. Krasemann S, Madore C, Cialic R, Baufeld C, Calcagno N, El Fatimy R, et al. The TREM2-APOE

11 Pathway Drives the Transcriptional Phenotype of Dysfunctional Microglia in Neurodegenerative Diseases.

12 Immunity. 2017;47(3):566-81 e9.

13 37. Keren-Shaul H, Spinrad A, Weiner A, Matcovitch-Natan O, Dvir-Szternfeld R, Ulland TK, et al. A

14 Unique Microglia Type Associated with Restricting Development of Alzheimer's Disease. Cell.

$15 \quad 2017 ; 169(7): 1276-90$ e17.

16 38. Kleinberger G, Brendel M, Mracsko E, Wefers B, Groeneweg L, Xiang X, et al. The FTD-like

17 syndrome causing TREM2 T66M mutation impairs microglia function, brain perfusion, and glucose

18 metabolism. EMBO J. 2017;36(13):1837-53.

19 39. Wang Y, Cella M, Mallinson K, Ulrich JD, Young KL, Robinette ML, et al. TREM2 lipid sensing

20 sustains the microglial response in an Alzheimer's disease model. Cell. 2015;160(6):1061-71.

21 40. Jay TR, Miller CM, Cheng PJ, Graham LC, Bemiller S, Broihier ML, et al. TREM2 deficiency

22 eliminates TREM2+ inflammatory macrophages and ameliorates pathology in Alzheimer's disease mouse

23 models. J Exp Med. 2015;212(3):287-95.

24 41. Yuan P, Condello C, Keene CD, Wang Y, Bird TD, Paul SM, et al. TREM2 Haplodeficiency in

25 Mice and Humans Impairs the Microglia Barrier Function Leading to Decreased Amyloid Compaction and

26 Severe Axonal Dystrophy. Neuron. 2016;90(4):724-39.

27 42. Painter MM, Atagi Y, Liu CC, Rademakers R, Xu H, Fryer JD, et al. TREM2 in CNS homeostasis

28 and neurodegenerative disease. Mol Neurodegener. 2015;10:43. 
1 43. Mazaheri F, Snaidero N, Kleinberger G, Madore C, Daria A, Werner G, et al. TREM2 deficiency

2 impairs chemotaxis and microglial responses to neuronal injury. EMBO Rep. 2017;18(7):1186-98.

3 44. Kotredes K. Data resource for manuscript - A multi-discipline phenotyping platform for late-onset

4 Alzheimer's disease employed on a novel, humanized APOEe4.Trem2*R47H mouse model [Internet]:

5 Synapse; 2020 [cited 2020. Available from: https://repo-

6 prod.prod.sagebase.org/repo/v1/doi/locate?id=syn23631984\&type=ENTITY.

$7 \quad 45 . \quad$ de Rezende LF, Rodrigues Lopes, M., Rey-López, J. P., Matsudo, V. K., \& Luiz, O. Sedentary

8 behavior and health outcomes: an overview of systematic reviews. PloS one. 2014;9(8):e105620.

9 46. Yan S, Fu, W., Wang, C., Mao, J., Liu, B., Zou, L., \& Lv, C. Association between sedentary

10 behavior and the risk of dementia: a systematic review and meta-analysis. Translational psychiatry.

$112020 ; 10(1): 12$.

12 47. Fenesi B, Fang, H., Kovacevic, A., Oremus, M., Raina, P., \& Heisz, J. J. Physical Exercise

13 Moderates the Relationship of Apolipoprotein E (APOE) Genotype and Dementia Risk: A Population-

14 Based Study. Journal of Alzheimer's disease : JAD. 2017;56(1):297-303.

15 48. Bilkei-Gorzo A. Genetic mouse models of brain ageing and Alzheimer's disease. Pharmacol Ther.

$16 \quad 2014 ; 142(2): 244-57$.

17 49. Cummings J, Lee G, Ritter A, Sabbagh M, Zhong K. Alzheimer's disease drug development

18 pipeline: 2020. Alzheimers Dement (N Y). 2020;6(1):e12050.

19 50. Cummings JL, Morstorf T, Zhong K. Alzheimer's disease drug-development pipeline: few

20 candidates, frequent failures. Alzheimers Res Ther. 2014;6(4):37.

21 51. Cheng-Hathaway PJ, Reed-Geaghan, E. G., Jay, T. R., Casali, B. T., Bemiller, S. M.,

22 Puntambekar, S. S., von Saucken, V. E., Williams, R. Y., Karlo, J. C., Moutinho, M., Xu, G., Ransohoff, R.

23 M., Lamb, B. T., \& Landreth, G. E. The Trem2 R47H variant confers loss-of-function-like phenotypes in

24 Alzheimer's disease. Molecular neurodegeneration. 2018;13(1).

25 52. Xiang X, Piers, T. M., Wefers, B., Zhu, K., Mallach, A., Brunner, B., Kleinberger, G., Song, W.,

26 Colonna, M., Herms, J., Wurst, W., Pocock, J. M., \& Haass, C. The Trem2 R47H Alzheimer's risk variant

27 impairs splicing and reduces Trem2 mRNA and protein in mice but not in humans. Molecular

28 neurodegeneration. 2018;13(1):49. 
1 53. Cheng Q, Danao, J., Talreja, S., Wen, P., Yin, J., Sun, N., Li, C. M., Chui, D., Tran, D., Koirala,

2 S., Chen, H., Foltz, I. N., Wang, S., \& Sambashivan, S. TREM2-activating antibodies abrogate the

3 negative pleiotropic effects of the Alzheimer's disease variant Trem2R47H on murine myeloid cell

4 function. The Journal of biological chemistry. 2018;293(32):12620-33.

5 54. Yamazaki Y, Zhao N, Caulfield TR, Liu CC, Bu G. Apolipoprotein E and Alzheimer disease:

6 pathobiology and targeting strategies. Nat Rev Neurol. 2019;15(9):501-18.

7 55. Mayeux R, Saunders AM, Shea S, Mirra S, Evans D, Roses AD, et al. Utility of the apolipoprotein

8 E genotype in the diagnosis of Alzheimer's disease. Alzheimer's Disease Centers Consortium on

9 Apolipoprotein E and Alzheimer's Disease. N Engl J Med. 1998;338(8):506-11.

10 56. Knouff C, Hinsdale ME, Mezdour H, Altenburg MK, Watanabe M, Quarfordt SH, et al. Apo E

11 structure determines VLDL clearance and atherosclerosis risk in mice. J Clin Invest. 1999;103(11):1579-

1286.

13 57. Abdelhafiz AH, Rodriguez-Manas L, Morley JE, Sinclair AJ. Hypoglycemia in older people - a less

14 well recognized risk factor for frailty. Aging Dis. 2015;6(2):156-67.

15 58. Zhao N, Ren Y, Yamazaki Y, Qiao W, Li F, Felton LM, et al. Alzheimer's Risk Factors Age, APOE

16 Genotype, and Sex Drive Distinct Molecular Pathways. Neuron. 2020;106(5):727-42 e6.

17 59. Murray J, Tsui WH, Li Y, McHugh P, Williams S, Cummings M, et al. FDG and Amyloid PET in

18 Cognitively Normal Individuals at Risk for Late-Onset Alzheimer's Disease. Adv J Mol Imaging.

19 2014;4(2):15-26.

20 60. Roher AE, Debbins JP, Malek-Ahmadi M, Chen K, Pipe JG, Maze S, et al. Cerebral blood flow in

21 Alzheimer's disease. Vasc Health Risk Manag. 2012;8:599-611.

22 61. Thambisetty M, Beason-Held L, An Y, Kraut MA, Resnick SM. APOE epsilon4 genotype and 23 longitudinal changes in cerebral blood flow in normal aging. Arch Neurol. 2010;67(1):93-8.

24 62. Fox JG, Barthold SW, Davisson MT, Newcomer CE, Quimby FW, Smith AL. In: Fox JG, Davisson

25 MT, Quimby FW, Barthold SW, Newcomer CE, Smith AL, editors. The Mouse in Biomedical Research

26 (Second Edition). Burlington: Academic Press; 2007. p. xv-xvi. 
1 63. Sukoff Rizzo SJ, Anderson LC, Green TL, McGarr T, Wells G, Winter SS. Assessing Healthspan

2 and Lifespan Measures in Aging Mice: Optimization of Testing Protocols, Replicability, and Rater

3 Reliability. Curr Protoc Mouse Biol. 2018;8(2):e45.

4 64. Bogue MA, Philip VM, Walton DO, Grubb SC, Dunn MH, Kolishovski G, et al. Mouse Phenome

5 Database: a data repository and analysis suite for curated primary mouse phenotype data. Nucleic Acids

6 Res. 2020;48(D1):D716-D23.

7 65. Fitz NF, Wolfe CM, Playso BE, Biedrzycki RJ, Lu Y, Nam KN, et al. Trem2 deficiency differentially

8 affects phenotype and transcriptome of human APOE3 and APOE4 mice. Mol Neurodegener.

$9 \quad 2020 ; 15(1): 41$.

10 66. Jendresen C, Arskog V, Daws MR, Nilsson LN. The Alzheimer's disease risk factors

11 apolipoprotein E and TREM2 are linked in a receptor signaling pathway. J Neuroinflammation.

$12 \quad 2017 ; 14(1): 59$.

13 67. McGeer PL, Walker DG, Pitas RE, Mahley RW, McGeer EG. Apolipoprotein E4 (ApoE4) but not

14 ApoE3 or ApoE2 potentiates beta-amyloid protein activation of complement in vitro. Brain Res.

$15 \quad 1997 ; 749(1): 135-8$.

16 68. Wan YW, Al-Ouran R, Mangleburg CG, Perumal TM, Lee TV, Allison K, et al. Meta-Analysis of

17 the Alzheimer's Disease Human Brain Transcriptome and Functional Dissection in Mouse Models. Cell

18 Rep. 2020;32(2):107908.

19 69. Pandey RS, Graham L, Uyar A, Preuss C, Howell GR, Carter GW. Genetic perturbations of

20 disease risk genes in mice capture transcriptomic signatures of late-onset Alzheimer's disease. Mol

21 Neurodegener. 2019;14(1):50.

22 70. Kanekiyo T, Xu H, Bu G. ApoE and Abeta in Alzheimer's disease: accidental encounters or 23 partners? Neuron. 2014;81(4):740-54.

24 71. Parhizkar S, Arzberger T, Brendel M, Kleinberger G, Deussing M, Focke C, et al. Loss of TREM2

25 function increases amyloid seeding but reduces plaque-associated ApoE. Nat Neurosci. 2019;22(2):191-

26204.

27 72. Zhao Y, Wu X, Li X, Jiang LL, Gui X, Liu Y, et al. TREM2 Is a Receptor for beta-Amyloid that

28 Mediates Microglial Function. Neuron. 2018;97(5):1023-31 e7. 
1 73. Kober DL, Stuchell-Brereton MD, Kluender CE, Dean HB, Strickland MR, Steinberg DF, et al.

2 Functional insights from biophysical study of TREM2 interactions with apoE and Abeta1-42. Alzheimers

3 Dement. 2020.

4 74. McQuade A, Kang YJ, Hasselmann J, Jairaman A, Sotelo A, Coburn M, et al. Gene expression

5 and functional deficits underlie TREM2-knockout microglia responses in human models of Alzheimer's

6 disease. Nat Commun. 2020;11(1):5370.

7 75. Kober DL, Alexander-Brett JM, Karch CM, Cruchaga C, Colonna M, Holtzman MJ, et al.

8 Neurodegenerative disease mutations in TREM2 reveal a functional surface and distinct loss-of-function

9 mechanisms. Elife. 2016;5.

10 76. Preuss C, Pandey R, Piazza E, Fine A, Uyar A, Perumal T, et al. A novel systems biology

11 approach to evaluate mouse models of late-onset Alzheimer's disease. Mol Neurodegener.

$12 \quad 2020 ; 15(1): 67$.

13 77. Yu CH, Wang T, Sun YE, Yao SL, Tian JH, Yin DY. [Fluorine-18 fluorodeoxyglucose uptake in

14 patients with benign pulmonary nodules]. Zhonghua Wai Ke Za Zhi. 2006;44(2):90-2.

15 78. Green MA. A potential copper radiopharmaceutical for imaging the heart and brain: copper-

16 labeled pyruvaldehyde bis(N4-methylthiosemicarbazone). Int J Rad Appl Instrum B. 1987;14(1):59-61.

17 79. Mathias CJ, Welch MJ, Raichle ME, Mintun MA, Lich LL, McGuire AH, et al. Evaluation of a

18 potential generator-produced PET tracer for cerebral perfusion imaging: single-pass cerebral extraction

19 measurements and imaging with radiolabeled Cu-PTSM. J Nucl Med. 1990;31(3):351-9.

20 80. Algin O, Ozmen E. Heavily T2W 3D-SPACE images for evaluation of cerebrospinal fluid

21 containing spaces. Indian J Radiol Imaging. 2012;22(1):74-5.

22 81. Sokoloff L. Relation between physiological function and energy metabolism in the central nervous 23 system. J Neurochem. 1977;29(1):13-26.

24 82. Mathias CJ, Welch MJ, Green MA, Diril H, Meares CF, Gropler RJ, et al. In vivo comparison of 25 copper blood-pool agents: potential radiopharmaceuticals for use with copper-62. J Nucl Med.

26 1991;32(3):475-80. 
1 83. Frese T, Rouze NC, Bouman CA, Sauer K, Hutchins GD. Quantitative comparison of FBP, EM,

2 and Bayesian reconstruction algorithms for the IndyPET scanner. IEEE Trans Med Imaging.

$3 \quad 2003 ; 22(2): 258-76$.

4 84. Soon KH, Farouque HM, Chaitowitz I, Cox N, Selvanayagam JB, Zakhem B, et al. Discrepancy

5 between computed tomography coronary angiography and selective coronary angiography in the pre-

6 stenting assessment of coronary lesion length. Australas Radiol. 2007;51(5):440-5.

7 85. Franklin KBJ, Paxinos G. Paxinos and Franklin's The mouse brain in stereotaxic coordinates.

8 Fourth edition. ed. Amsterdam: Academic Press, an imprint of Elsevier; 2013. 1 volume (unpaged) p.

9 86. Territo PR, Meyer JA, Peters JS, Riley AA, McCarthy BP, Gao M, et al. Characterization of (11)C-

10 GSK1482160 for Targeting the P2X7 Receptor as a Biomarker for Neuroinflammation. J Nucl Med.

$112017 ; 58(3): 458-65$.

12 87. Studholme C, Hill DL, Hawkes DJ. Automated three-dimensional registration of magnetic

13 resonance and positron emission tomography brain images by multiresolution optimization of voxel

14 similarity measures. Med Phys. 1997;24(1):25-35.

15 88. Bolger AM, Lohse M, Usadel B. Trimmomatic: a flexible trimmer for Illumina sequence data.

16 Bioinformatics. 2014;30(15):2114-20.

17 89. Dobin A, Davis CA, Schlesinger F, Drenkow J, Zaleski C, Jha S, et al. STAR: ultrafast universal

18 RNA-seq aligner. Bioinformatics. 2013;29(1):15-21.

19 90. Li B, Dewey CN. RSEM: accurate transcript quantification from RNA-Seq data with or without a

20 reference genome. BMC Bioinformatics. 2011;12(1):323.

21 91. Anders S, Pyl PT, Huber W. HTSeq-a Python framework to work with high-throughput

22 sequencing data. Bioinformatics. 2015;31(2):166-9.

23 92. Love Ml, Huber W, Anders S. Moderated estimation of fold change and dispersion for RNA-seq

24 data with DESeq2. Genome Biology. 2014;15(12):550.

25 93. Yu G, Wang L-G, Han Y, He Q-Y. clusterProfiler: an R Package for Comparing Biological Themes

26 Among Gene Clusters. OMICS : a Journal of Integrative Biology. 2012;16(5):284-7.

27 94. De Jager PL, Ma Y, McCabe C, Xu J, Vardarajan BN, Felsky D, et al. A multi-omic atlas of the

28 human frontal cortex for aging and Alzheimer's disease research. Scientific data. 2018;5:180142-. 
1 95. Ritchie ME, Phipson B, Wu D, Hu Y, Law CW, Shi W, et al. limma powers differential expression

2 analyses for RNA-sequencing and microarray studies. Nucleic acids research. 2015;43(7):e47-e.

3 96. Percie du Sert N, Hurst V, Ahluwalia A, Alam S, Avey MT, Baker M, et al. The ARRIVE guidelines

4 2.0: Updated guidelines for reporting animal research. PLoS Biol. 2020;18(7):e3000410.

7 Acknowledgements

8 The authors would like to acknowledge the members of the Jackson Laboratory Center for Biometrics

9 Analysis for their efforts toward the behavioral phenotyping of these animals, the Jackson Laboratory's

10 Genetic Engineering Technologies Scientific Service for strain development, the Jackson Laboratory's

11 Clinical Assessment Services for tissue and blood chemistry analysis, and the Jackson Laboratory's

12 Genome Technologies group for RNA-sequencing and Nanostring assays.

\section{Author contributions}

15 K.P.K: Data curation, Formal analysis, Investigation, Methodology, Writing; A.O.: Conceptualization, Data 16 curation, Formal analysis, Investigation, Methodology, Writing; $\quad$ R.P.: $\quad$ Data curation, Formal

17 analysis, Investigation, Methodology, Writing; $\quad$ P.B.: $\quad$ Formal $\quad$ analysis, Investigation; $\quad$ D.G.:

18 Investigation, Methodology; H.W.: Data curation, Formal analysis, Investigation, Methodology; A.U.: Data 19 curation, Formal analysis, Investigation; R.O.: Investigation; S.O.: Investigation; C.I.: Investigation; D.B.:

20 Investigation; M.B.: Investigation; Z.C.: Investigation; K.E.F.: Investigation; B.A.L.:

21 Methodology, Investigation; L.M.M.: Methodology, Investigation; S.J.S.R.: Conceptualization, Data

22 curation, Investigation, Methodology, Writing; P.R.T.: Conceptualization, Data

23 curation, Investigation, Methodology, Writing; $\quad$ G.W.C.: Conceptualization, Data

24 curation, Methodology, Writing; $\quad$ M.S.: Conceptualization, Methodology, Writing; $\quad$ B.T.L.:

25 Conceptualization, Methodology, Writing; G.R.H.: Conceptualization, Investigation, Methodology, Writing. 26

\section{ARRIVE guidelines statement}


1 In accordance with ARRIVE (Animal Research: Reporting of In Vivo Experiments) guidelines, design and

2 description of experimental animal cohorts is provided to ensure scientific rigor and reproducibility (96).

3 (https://arriveguidelines.org/)

5 Data availability statement

6 The B6.APOE4.Trem2*R47H (LOAD1) data sets are available via the AD Knowledge Portal

7 (https://adknowledgeportal.org). The AD Knowledge Portal is a platform for accessing data, analyses, and

8 tools generated by the Accelerating Medicines Partnership (AMP-AD) Target Discovery Program and other

9 National Institute on Aging (NIA)-supported programs to enable open-science practices and accelerate

10 translational learning. The data, analyses and tools are shared early in the research cycle without a

11 publication embargo on secondary use. Data is available for general research use according to the following

12 requirements for data access and data attribution

13 (https://adknowledgeportal.org/DataAccess/Instructions).

14 For access to content described in this manuscript see: https://doi.org/10.7303/syn23631984

16 Ethics approval

17 No human subjects or data was used in this study. All experiments involving mice were approved by the

18 Animal Care and Use Committee at The Jackson Laboratory in accordance with guidelines set out in The

19 Eighth Edition of the Guide for the Care and Use of Laboratory Animals. All euthanasia used methods were 20 approved by the American Veterinary Medical Association.

\section{Consent for publication}

23 Not applicable

\section{Competing interests statement}

26 The authors declare that they have no competing interests

\section{$28 \quad$ Funding}


1 This work was supported primarily by The Jackson Laboratory startup funds to GRH, and also National

2 institute of General Medical Sciences T32HD007065 (KDO), National Institute on Aging RF1AG055104

3 (GRH) and National Institute on Aging U54AG054345 (MODEL-AD). The funders had no role in study

4 design, data collection and analysis, decision to publish, or preparation of the manuscript.

7 LIST OF ABBREVIATIONS

8 AD: Alzheimer's disease

9 ADGC: Alzheimer's Disease Genetics Consortium

10 ADNI: Alzheimer's Disease Neuroimaging Initiative

11 ADSP: Alzheimer's Disease Sequencing Project

12 AMP-AD: Accelerating Medicines Partnership - Alzheimer's Disease

13 B6: C57BL/6J

14 BBB: Blood-brain barrier

15 CNS: Central nervous system

16 DEG: Differentially expressed genes

17 FP: frontal pole brain regions

18 HDL: high-density lipoproteins

19 IGAP: International Genomics of Alzheimer's Project

20 LOAD: Late-onset Alzheimer's disease

21 LDL: low-density lipoproteins

$22 M^{2}$ OVE-AD: Molecular Mechanisms of the Vascular Etiology of Alzheimer's Disease

23 MRI: Magnetic resonance imaging

24 PET: Positron emission tomography

25 PHG: parahippocampal gyrus

26 ROS/MAP: Religious Orders Study and Memory and Aging Project 
FIGURE LEGENDS

Figure 1. Age is primary factor driving increases in measures of frailty. Cross-sectional cohorts of young and old mice inspected for measures of physical well-being, or frailty $(A, B)$, including body weight (C,D). Post-mortem, non-fasted, blood biochemistry analysis provided serum levels of cholesterol, lipoproteins, lipids, and glucose (E-L). Age-dependent differences within genotype determined by ANOVA: ${ }^{*} p<0.05 ;{ }^{* *} p<0.01 ;{ }^{* * *} p<0.001$. All alleles expressed were homozygous.

Figure 2. Behavioral testing of $A P O E 4 . T r e m 2{ }^{\star} R 47 H$ mice to identify functional neurophenotype.

9 Performance of young and aged mice carrying combinations of $A P O E \varepsilon 4$ and $T r e m 2^{*} R 47 H$ alleles were 10 evaluated for measures of coordination (rotarod; A,B), locomotor activity (open field; C-F), exploratory drive

11 (open field; G,H), and spatial working memory (spontaneous alternation in Y-maze; I-L). Age-dependent 12 differences within genotype determined by ANOVA: ${ }^{*} p<0.05 ;{ }^{* *} p<0.01 ;{ }^{* * *} p<0.001$. All alleles expressed 13 were homozygous.

15 Figure 3. Variations in regional glucose metabolism due to expression of $A P O E \varepsilon 4$ and $T r e m 2{ }^{\star} R 47 H$.

16 Positron emission tomography (PET; red scale) of radioactive 18F-FDG marker was used to measure tissue

17 glucose uptake, guided by magnetic resonance imaging (MRI) (black and white) mapping to brain regions 18 of interest, indicated by bregma coordinates (far left) (A). Intensity of PET signal in brains regions, 19 normalized to cerebellum, are quantified in B,C. Post-mortem autoradiography of coronal brain tissue is 20 represented in A (rainbow; far right). Genotype-dependent differences determined by ANOVA: * $p<0.05$; $21{ }^{* *} p<0.01 ;{ }^{* * *} p<0.001$. All alleles expressed were homozygous.

23 Figure 4. In vivo neuroimaging reveals differences in regional perfusion driven by expression of 24 risk alleles. Positron emission tomography (PET; red scale) of radioactive 64Cu-PTSM marker was used 25 to measure tissue perfusion, guided by magnetic resonance imaging (MRI) (black and white) mapping to 26 brain regions of interest, indicated by bregma coordinates (far left) (A). Intensity of PET signal in brains 27 regions, normalized to cerebellum, are quantified in B,C. Post-mortem autoradiography of coronal brain 
tissue is represented in A (rainbow; far right). Genotype-dependent differences determined by ANOVA: ${ }^{*} p<0.05 ;{ }^{* *} p<0.01 ;{ }^{* * *} p<0.001$. All alleles expressed were homozygous.

Figure 5. Neuropathological analysis of cortex and hippocampus. Hematoxylin and eosin (H\&E), luxol fast blue/cresyl violet (LFB/CV), and Prussian blue staining tissue staining protocols (A) to identify anatomical changes in the cortex and hippocampus of young and aged male mice. Thioflavin S (Thios) and four combinations of known neuropathological markers of LOAD in aged male mice (B); 5xFAD included as amyloidogenic positive control. All alleles expressed were homozygous.

Figure 6. Overview of brain transcriptome. Expression levels of mouse Apoe (A), human $A P O E(\mathrm{~B})$, and

11 mouse Trem2 (C) genes in the B6.APOE4, B6.Trem2* $R 47 H, B 6 . A P O E 4 . T r e m 2{ }^{*} R 47 H$ and C57BL/6J mice

12 at 4, 8, 12 and 24 months in both sexes. Principal component analysis (PCA) of RNA-Seq transcriptomics

13 data from all 234 samples (D). The percent of variation explained by each principal component is displayed

14 on the corresponding axis. Female and male samples are represented as circles and triangles, respectively.

15 Genotypes are shown by different colors and increasing size of points correspond to increasing age of mice $16(4,8,12$, and 24 months respectively). All alleles expressed were homozygous.

18 Figure 7. KEGG pathways enrichment analysis. Significantly enriched KEGG pathways $(p<0.05)$ in the 19 downregulated and upregulated list of genes across mouse models at different ages for both sexes. All 20 alleles expressed were homozygous.

\section{TABLES}

24 Table 1

\begin{tabular}{|l|l|l|l|l|l|l|l|}
\hline $\begin{array}{l}\text { Common } \\
\text { name }\end{array}$ & $\begin{array}{l}\text { JAX } \\
\text { stock } \\
\#\end{array}$ & Strain & Background & $\begin{array}{l}\text { Gene } \\
\text { location }\end{array}$ & $\begin{array}{l}\text { Allele } \\
\text { name }\end{array}$ & Allele type & Additional considerations \\
\hline B6 & 000664 & C57BL/6J & - & - & - & - & - \\
\hline Trem2*R47H & 027918 & $\begin{array}{l}\text { C57BL/6J- } \\
\text { Trem2em1Adiuj/J }\end{array}$ & C57BL/6J & $\begin{array}{l}\text { Chr17:4834 } \\
6401- \\
48352276\end{array}$ & $\begin{array}{l}\text { Trem2 } \\
\text { R47H KI KI }\end{array}$ & $\begin{array}{l}\text { Cas9 endonuclease- } \\
\text { mediated } \\
\text { (humanized } \\
\text { sequence) }\end{array}$ & $\begin{array}{l}\text { - Two silent mutations (lysine } \\
\text { AAG }>\text { AAA and alanine } \\
\text { GCC }>\text { GCA) into Trem2 }\end{array}$ \\
\hline
\end{tabular}




\begin{tabular}{|c|c|c|c|c|c|c|c|}
\hline & & & & & & & $\begin{array}{l}\text { - R47H mutation also introduces } \\
\text { a cryptic splice acceptor site in } \\
\text { exon } 2 \text {, creating a novel splice } \\
\text { variant with a deletion of } 119 \mathrm{bp} \\
\text { at the } 5 \text { ' end of exon } 2 \text {. }\end{array}$ \\
\hline APOE4 & 027894 & $\begin{array}{l}\text { B6(SJL)- } \\
\text { Apoe }^{\text {tm1.1(APOE } 4) \text { Adiuj/J }}\end{array}$ & C57BL/6J & $\begin{array}{l}\text { Chr7:19696 } \\
109- \\
19699188\end{array}$ & $\begin{array}{l}\text { APOE4 } \\
\mathrm{KI}\end{array}$ & $\begin{array}{l}\text { FRT site flanked } \\
\text { PGK- neo cassette } \\
\text { targeted mutation } \\
\text { (gene replacement) }\end{array}$ & $\begin{array}{l}\text { - Exons } 2,3 \text { and a majority of } \\
\text { exon } 4 \text { of the mouse Apoe gene } \\
\text { were replaced by exons } 2,3 \text {, } \\
\text { and } 4 \text { of the human } A P O E \text { gene } \\
\text { sequence (including a portion of } \\
\text { the } 3 \text { ' UTR sequence) } \\
\text { - Expression of FLP } \\
\text { recombinase was used to } \\
\text { remove the FRT site flanked } \\
\text { PGK-neo cassette and } \\
\text { subsequently backcrossed to } \\
\text { remove FLP recombinase }\end{array}$ \\
\hline $\begin{array}{l}\text { APOE4. } \\
\text { Trem2*R47H }\end{array}$ & 028709 & $\begin{array}{l}\text { B6(SJL)- } \\
\text { Apoe }{ }^{\text {tm1.1(APOE }} \text { 4)Adiuj } \\
\text { Trem2 } 2 \text { em1Adiuj/J }\end{array}$ & C57BL/6J & (see above) & $\begin{array}{l}\text { (see } \\
\text { above) }\end{array}$ & (see above) & (see above) \\
\hline
\end{tabular}

2 Table 1. Description of novel mouse strains expressing human LOAD risk alleles. Mouse strains

3 expressing $T r e m 2^{*} R 47 H$ and $A P O E \varepsilon 4$ human late-onset Alzheimer Disease risk factors, alone or in

4 combination, developed on the C57BL/6J background and distributed by The Jackson Laboratory

$5 \quad$ [https://www.jax.org/mouse-search]. 


\section{Figures}

A.

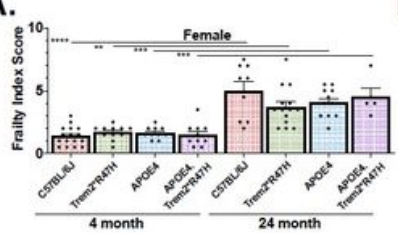

B.

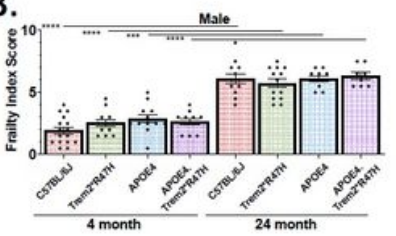

E.
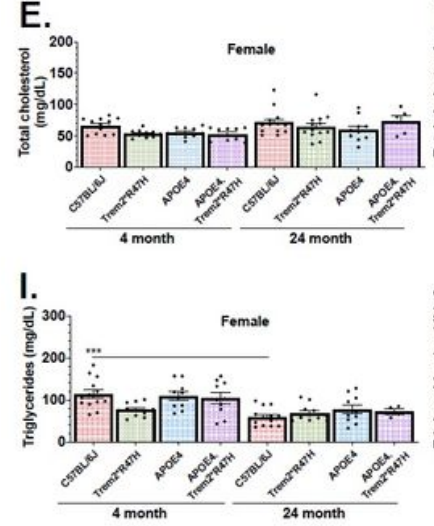
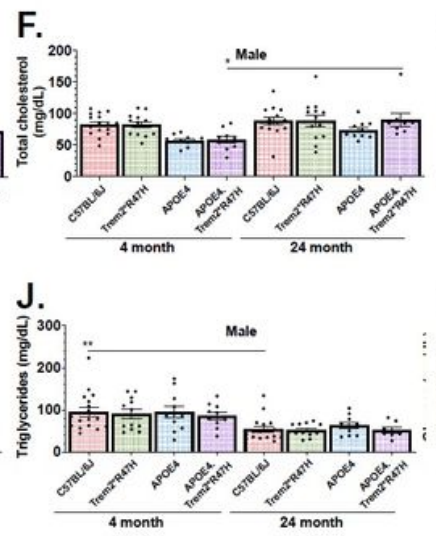
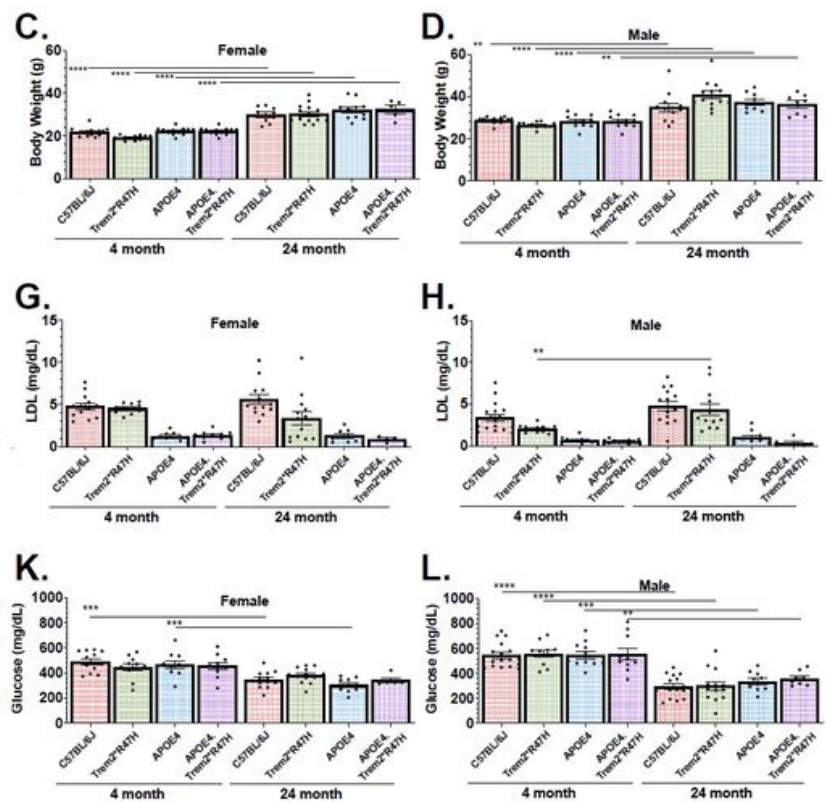

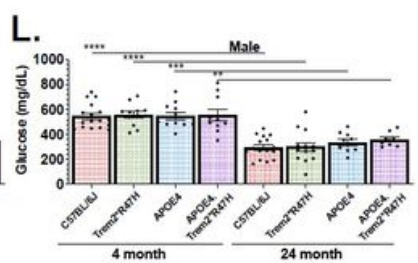

\section{Figure 1}

Age is primary factor driving increases in measures of frailty. Cross-sectional cohorts of young and old mice inspected for measures of physical well-being, or frailty $(A, B)$, including body weight $(C, D)$. Postmortem, non-fasted, blood biochemistry analysis provided serum levels of cholesterol, lipoproteins, lipids, and glucose (E-L). Age-dependent differences within genotype determined by ANOVA: ${ }^{*} p<0.05 ;{ }^{* *} \mathrm{p}<0.01$; $* \star * p<0.001$. All alleles expressed were homozygous. 


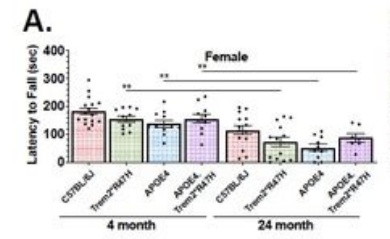

E.
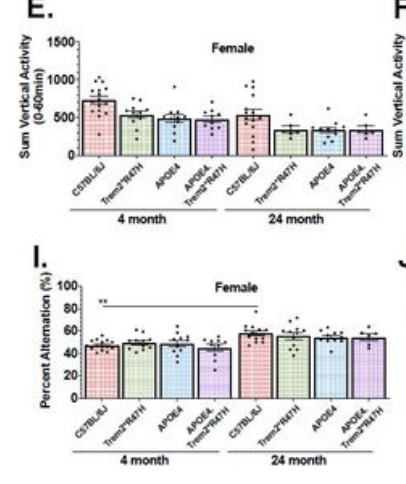

B.

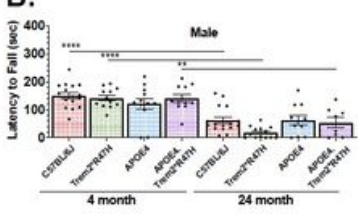

F.
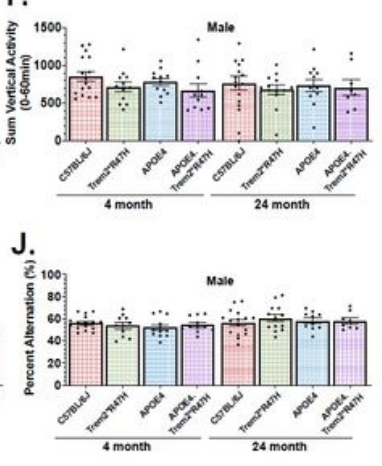

c.

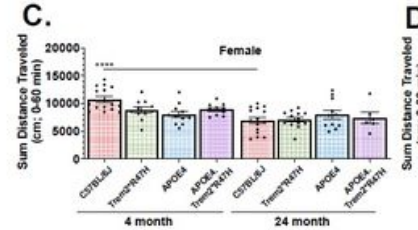

G.

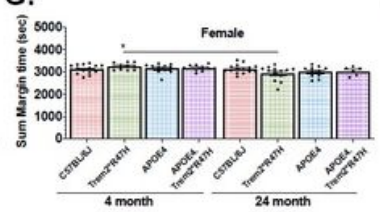

$\mathrm{K}$.

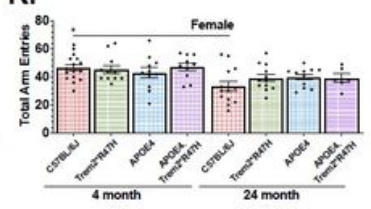

H.
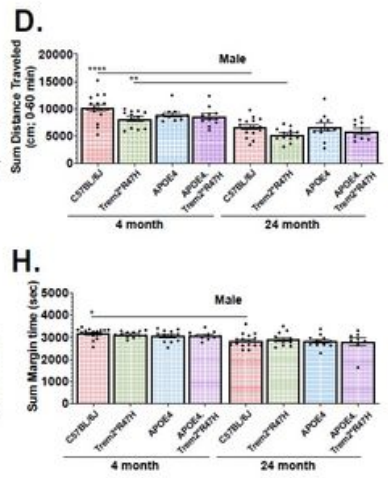

L.

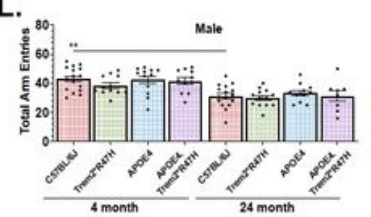

\section{Figure 2}

Behavioral testing of APOE4.Trem2*R47H mice to identify functional neurophenotype. Performance of young and aged mice carrying combinations of APOE 4 and Trem $2{ }^{*} \mathrm{R} 47 \mathrm{H}$ alleles were evaluated for measures of coordination (rotarod; $A, B$ ), locomotor activity (open field; C-F), exploratory drive (open field; $\mathrm{G}, \mathrm{H}$ ), and spatial working memory (spontaneous alternation in Y-maze; I-L). Age-dependent differences within genotype determined by ANOVA: ${ }^{*} p<0.05 ;{ }^{* \star} p<0.01 ;{ }^{* *} p<0.001$. All alleles expressed were homozygous. 

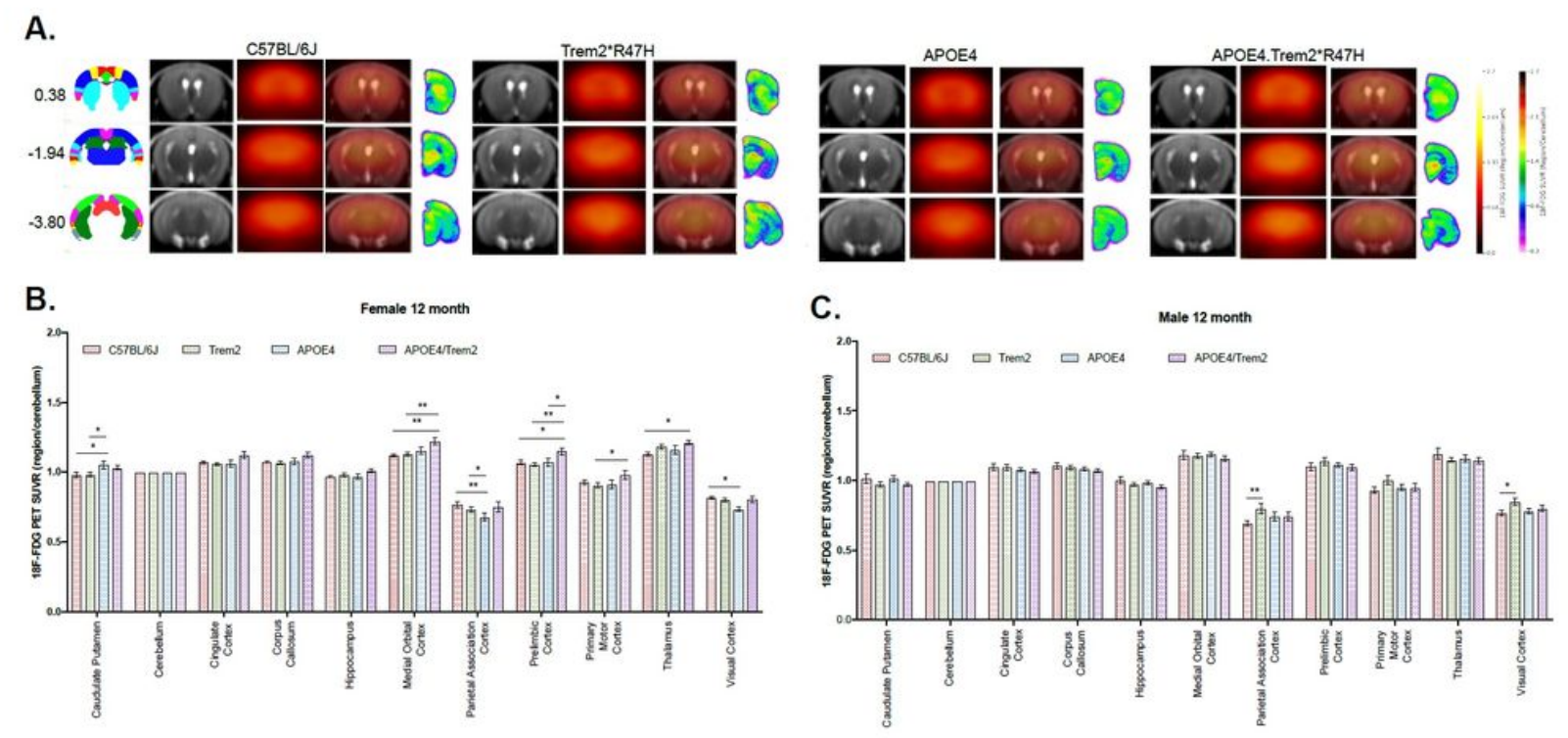

\section{Figure 3}

Variations in regional glucose metabolism due to expression of APOE\&4 and Trem2*R47H. Positron emission tomography (PET; red scale) of radioactive 18F-FDG marker was used to measure tissue glucose uptake, guided by magnetic resonance imaging (MRI) (black and white) mapping to brain regions of interest, indicated by bregma coordinates (far left) (A). Intensity of PET signal in brains regions, normalized to cerebellum, are quantified in B,C. Post-mortem autoradiography of coronal brain tissue is represented in A (rainbow; far right). Genotype-dependent differences determined by ANOVA: ${ }^{*} \mathrm{p}<0.05$; $\star * p<0.01 ; * * * p<0.001$. All alleles expressed were homozygous. 
A.

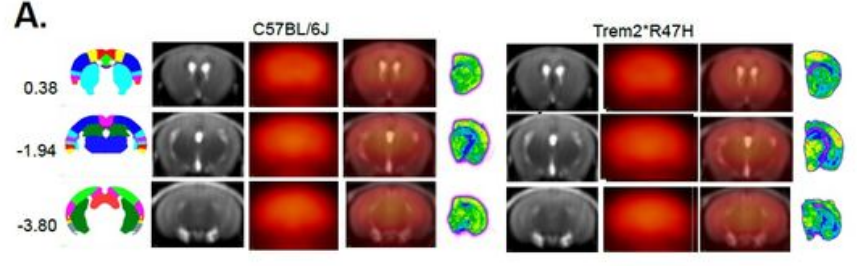

B.

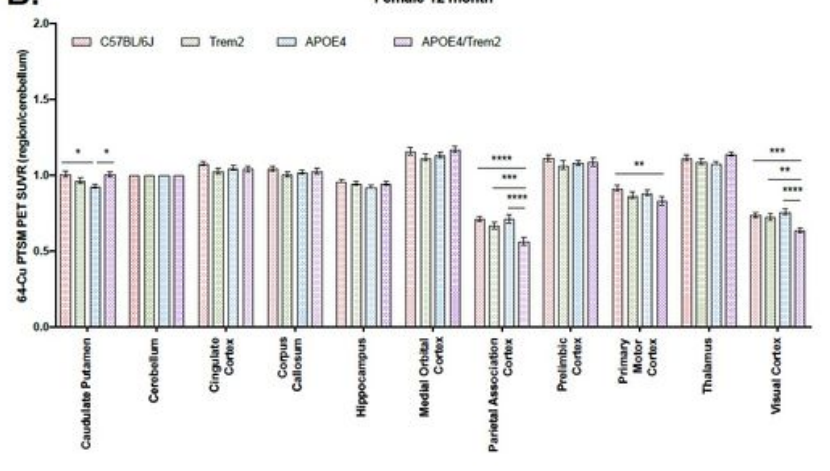

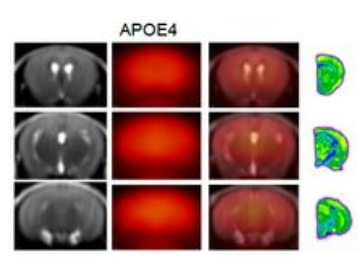

$\mathrm{C}_{\text {is }}$

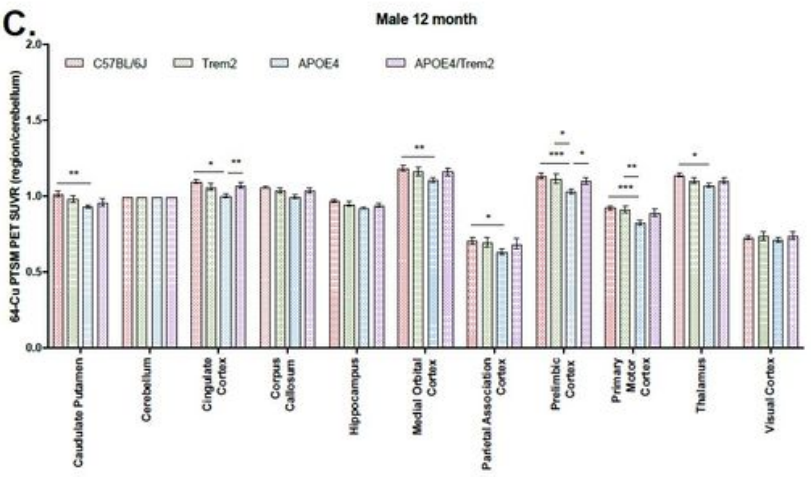

Figure 4

In vivo neuroimaging reveals differences in regional perfusion driven by expression of risk alleles. Positron emission tomography (PET; red scale) of radioactive 64Cu-PTSM marker was used to measure tissue perfusion, guided by magnetic resonance imaging (MRI) (black and white) mapping to brain regions of interest, indicated by bregma coordinates (far left) (A). Intensity of PET signal in brains regions, normalized to cerebellum, are quantified in B,C. Post-mortem autoradiography of coronal brain tissue is represented in A (rainbow; far right). Genotype-dependent differences determined by ANOVA: ${ }^{*} p<0.05 ;{ }^{* *} p<0.01 ;{ }^{* \star *} p<0.001$. All alleles expressed were homozygous. 
A.
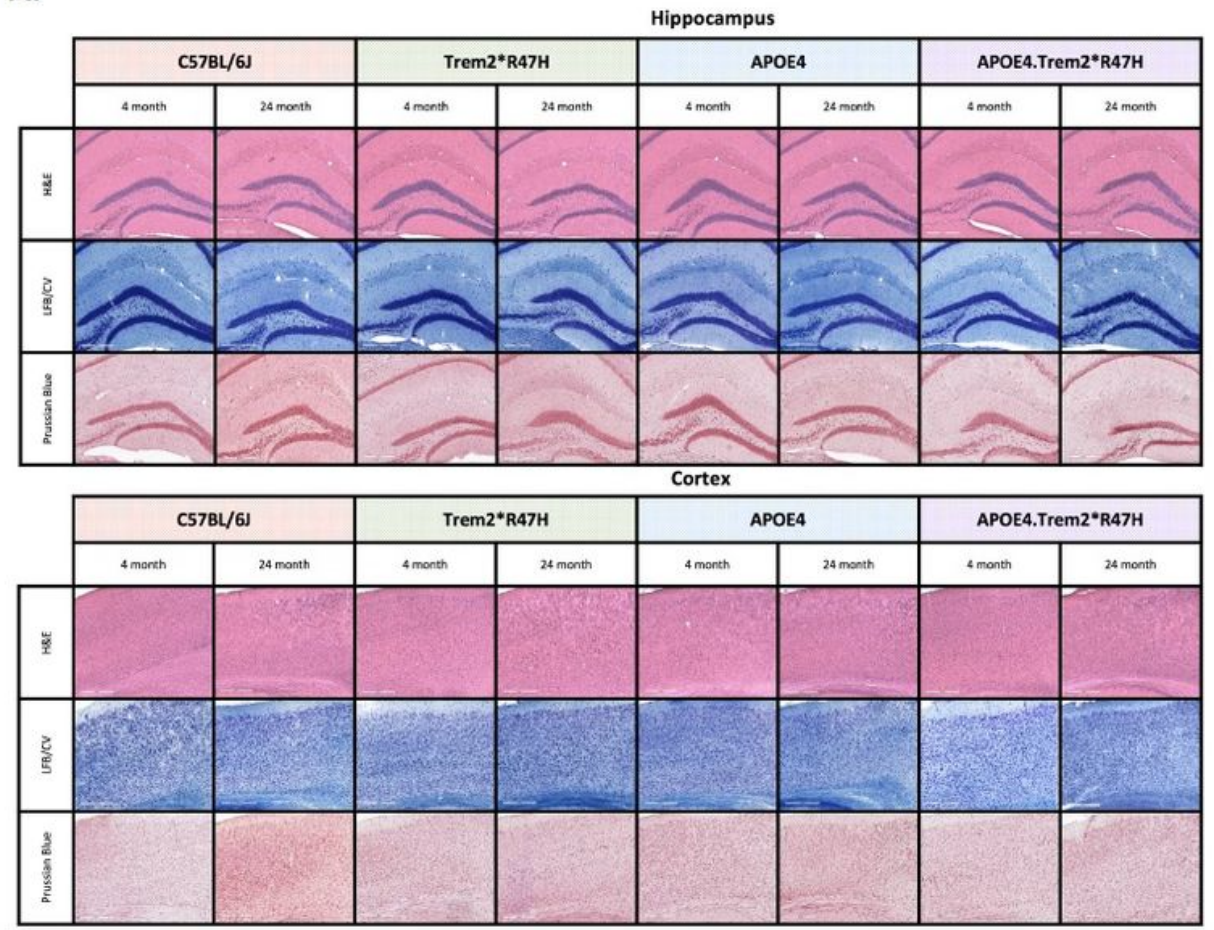

B.

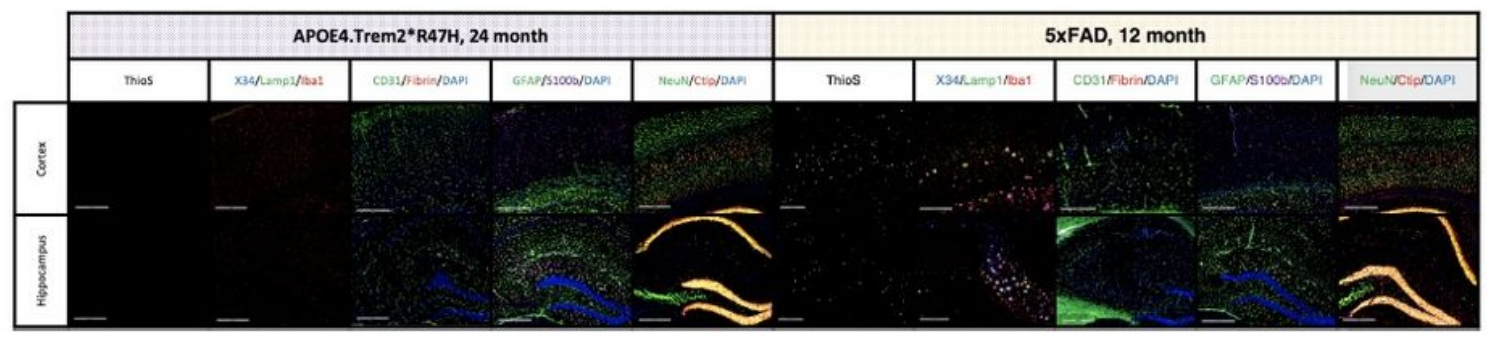

\section{Figure 5}

Neuropathological analysis of cortex and hippocampus. Hematoxylin and eosin (H\&E), luxol fast blue/cresyl violet (LFB/CV), and Prussian blue staining tissue staining protocols (A) to identify anatomical changes in the cortex and hippocampus of young and aged male mice. Thioflavin $S$ (Thios) and four combinations of known neuropathological markers of LOAD in aged male mice (B); 5xFAD included as amyloidogenic positive control. All alleles expressed were homozygous. 


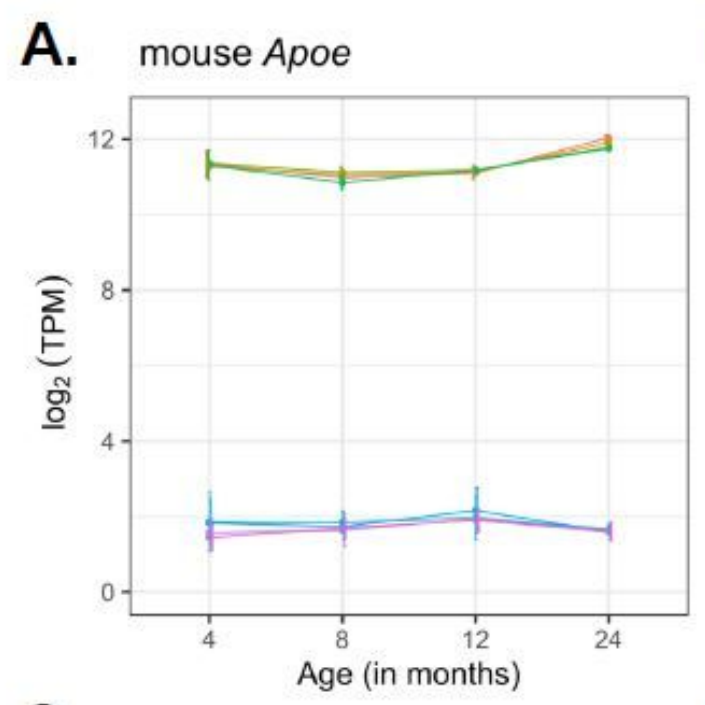

B. human $A P O E$
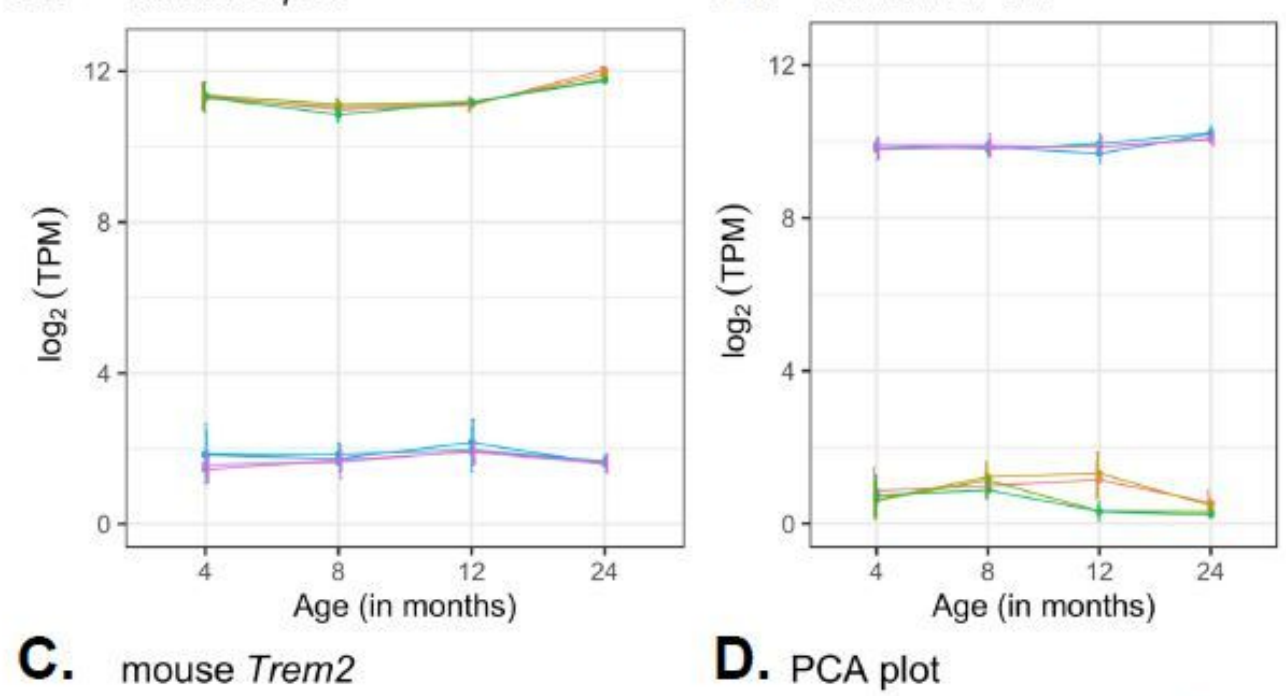

Group

- C57BL/6J Female

- C57BL/6J Male

- Trem2*R47H Male

- Trem2*R47H Female

- APOE4 Female

- APOE4 Male

- APOE4.Trem2*R47H Female

- APOE4.Trem2*R47H Male

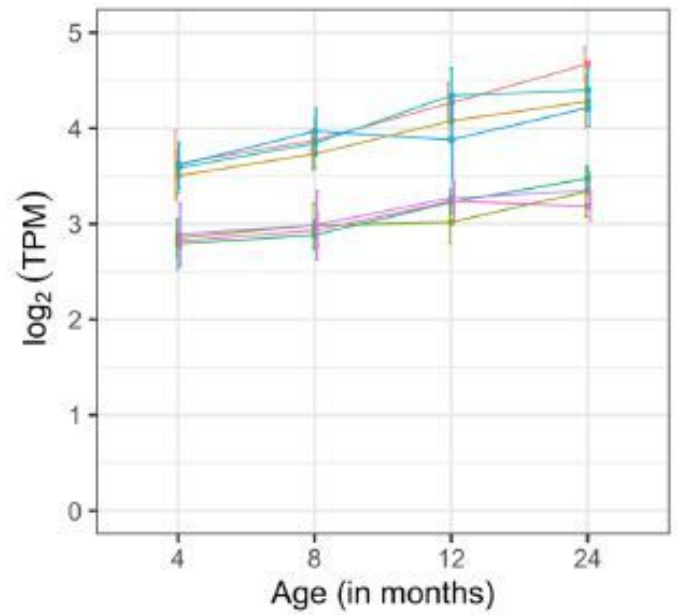

D. PCA plot

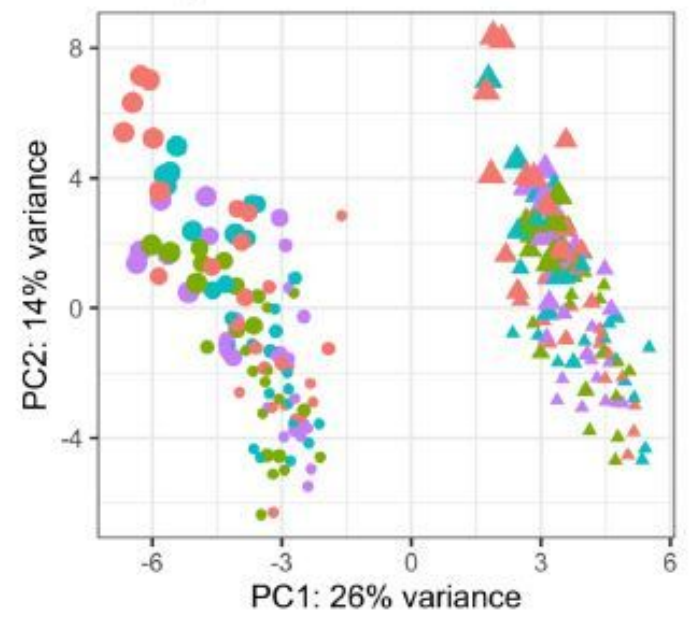

Sex

- Fernale

- Male

Age

- 4

- 8

12
24

Genotype

- C57BL/6J

- Trem2*R47H

- APOE4

- APOE4.Trem2*R47H

Figure 6

Overview of brain transcriptome. Expression levels of mouse Apoe (A), human APOE (B), and mouse Trem2 (C) genes in the B6.APOE4, B6.Trem2*R47H, B6.APOE4.Trem2*R47H and C57BL/6J mice at 4, 8, 12 and 24 months in both sexes. Principal component analysis (PCA) of RNA-Seq transcriptomics data from all 234 samples (D). The percent of variation explained by each principal component is displayed on the corresponding axis. Female and male samples are represented as circles and triangles, respectively. Genotypes are shown by different colors and increasing size of points correspond to increasing age of mice $(4,8,12$, and 24 months respectively). All alleles expressed were homozygous. 
Kegg pathway enrichment across mouse models

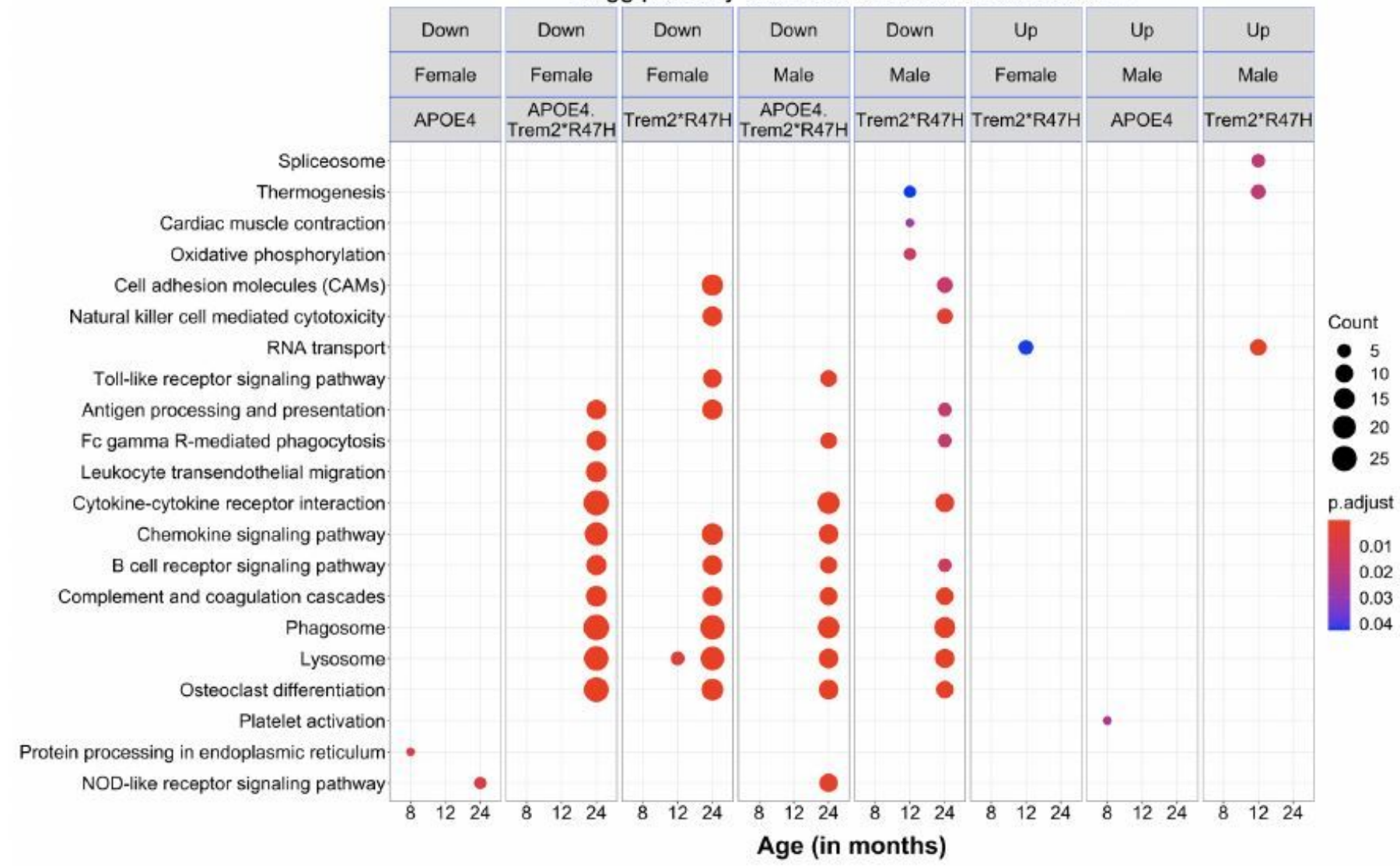

\section{Figure 7}

KEGG pathways enrichment analysis. Significantly enriched KEGG pathways $(p<0.05)$ in the downregulated and upregulated list of genes across mouse models at different ages for both sexes. All alleles expressed were homozygous.

\section{Supplementary Files}

This is a list of supplementary files associated with this preprint. Click to download.

- LOAD1figuresv4SupplementalFigures.pdf

- LOAD1Table1.pdf 